Investigaciones geográficas, $n .^{\circ} 45$ (2008) pp. 163-199 ISSN: 0213-4691
Instituto Universitario de Geografía Universidad de Alicante

\title{
EXTRANJERÍA Y DIFERENCIACIÓN RESIDENCIAL EN CANARIAS: \\ LA PERSPECTIVA DEL MICROANÁLISIS ESPACIAL ${ }^{1}$
}

\author{
Juan Manuel Parreño Castellano \\ Josefina Domínguez Mujica \\ Departamento de Geografía \\ Universidad de Las Palmas de Gran Canaria
}

\section{Introducción}

Desde hace unos treinta años viene desarrollándose en Geografía el análisis de los procesos de segregación espacial en relación con los grupos de población de procedencia exterior. Estos procesos generalmente afrontan realidades consolidadas, en ámbitos territoriales en los que se manifiestan situaciones de «guetización». En muy pocos casos se realizan estos estudios cuando el fenómeno migratorio tiene una escasa antigüedad. Éste es el caso de España, y particularmente de Canarias, zonas en las que la afluencia de población extranjera, en una dimensión significativa, es relativamente reciente, de no más de diez años. Por ello, el análisis del fenómeno en la realidad geográfica del archipiélago canario, un archipiélago español atlántico, de especialización turística, permite adelantarnos a las tendencias de futuro que comienzan a mostrarse y a reconocer si se están generando procesos territoriales semejantes al de otras zonas con dinámicas migratorias «maduras».

1. Este texto adelanta algunos resultados del proyecto de investigación: «Repercusiones socioeconómicas y territoriales de la vivienda de la población extranjera en Canarias» (PI042005/127), dirigido por Josefina Domínguez Mujica y financiado por la Dirección General de Universidades e Investigación de la Consejería de Educación, Cultura y Deportes del Gobierno de Canarias. 
Nuestro objetivo es desvelar los procesos de diferenciación social del espacio, en correspondencia con la reciente presencia de extranjeros. Se intenta caracterizar la dimensión espacial de un fenómeno migratorio que, en clara sintonía con su carácter reciente, intenso y dinámico, queda aún indefinido en muchas de sus facetas geográficas. Por ello, se hace necesario acudir a nuevos procedimientos de análisis que revelen algunas de sus características.

En los últimos años el Instituto Nacional de Estadística (España) y el Instituto Canario de Estadística (ISTAC) han comenzado a ofrecer la información demográfica, desagregada territorialmente hasta el nivel de distritos y secciones censales, tanto la que se obtuvo en el Censo de Población y Viviendas de 2001, como la que procede de la revisión de las cifras de población, a uno de enero de cada año (revisión padronal) desde 1998, lo que permite incorporar la perspectiva microescalar en los estudios geodemográficos. Por otra parte, los Sistemas de Información Geográfica brindan extraordinarias posibilidades de tratamiento estadístico para estos datos georreferenciados, todo lo cual convierte estos dos instrumentos en herramientas de una enorme utilidad para afrontar nuevas hipótesis de trabajo. Ellos son, en consecuencia, los que empleamos para documentar la diferenciación social del espacio de la extranjería: los modelos residenciales por nacionalidad y la medición de la segregación que la caracteriza.

Partimos de la premisa de que el mercado laboral, turístico y residencial debe determinar la distribución espacial de la población extranjera y, puesto que este fenómeno presenta un comportamiento territorial concentrado, el modelo residencial de la extranjería debiera ser también concentrado. Ahora bien, ¿cabe reconocer, además, un modelo concentrado en función de la nacionalidad? En segundo lugar, dadas las características migratorias y residenciales de Canarias (la intensidad de la reciente inmigración extranjera, la existencia de tejidos residenciales de baja calidad, el dinámico mercado de la segunda residencia para extranjeros...), debiera producirse cierta segregación, pero teniendo en cuenta la naturaleza de este proceso ¿podríamos llegar a medirlo estadísticamente?

En correspondencia con estos interrogantes, en este artículo se desarrolla una primera parte de carácter conceptual, en la que se sintetizan las principales líneas de análisis acerca de la diferenciación social del espacio, una segunda parte, de carácter metodológico, sobre las fuentes, las escalas y el método de trabajo desarrollado con la infraestructura de datos espaciales y una tercera parte en la que el análisis de la extranjería se aborda con una elección estadística de los espacios que definimos como espacios de la inmigración, en los 
que analizamos el comportamiento espacial por nacionalidades, así como los procesos de segregación.

Al margen de las conclusiones acerca de la extranjería en el ámbito concreto de Canarias, una región insular y distante del resto del Estado, en la frontera meridional de la Unión Europea, utilizamos un procedimiento de trabajo geodemográfico que consideramos de enorme interés en la caracterización de procesos que pueden desarrollarse en otros ámbitos espaciales.

\section{La diferenciación social del espacio a partir de la nacionalidad}

La preocupación por la distribución espacial de los grupos sociales se remonta a principios del siglo xx (Basset y Short, 1980). De este modo, los primeros estudios que abordaron los procesos de concentración y segregación y la diferenciación residencial del espacio fueron realizados por geógrafos y sociólogos vinculados a la ecología humana de la Escuela de Chicago. Este enfoque, basado en trasponer principios como los de competencia, dominancia o sucesión ecológica al comportamiento espacial, generó valiosos modelos de estructura urbana (Mackenzie, 1925).

Una preocupación más específica por la distribución espacial de los extranjeros hay que buscarla en los modelos deductivos de corte positivista que empezaron a surgir desde finales de los años cuarenta. En este sentido, los análisis de áreas sociales realizados por E. Shevky, M. Williams y W. Bell en Los Ángeles y San Francisco (Shevsky y Bell, 1955) introdujeron el estatus étnico o la movilidad migratoria como factores de diferenciación en áreas sociales homogéneas.

Tras estos primeros antecedentes, en décadas posteriores, proliferaron estudios en los que el análisis se centraba en los factores que explican la distribución territorial de los inmigrantes y extranjeros, en la medida en que se activaban los flujos migratorios internacionales y en que, poco a poco, nos encaminábamos hacia un mundo globalizado. El primer factor considerado fue el estatus económico o la posición en el mercado de trabajo, como así se manifestó, de manera temprana, en los enfoques factoriales y empíricos desarrollados en los setenta (Berry y Kasarda, 1977). En los años siguientes las aproximaciones se fueron diversificando y pronto surgirían enfoques culturalistas que pretendían relacionar los modelos residenciales y las condiciones de vivienda con las características culturales de los diferentes grupos nacionales.

Una tercera línea de aproximación es la que interpreta las estructuras territoriales a partir del mercado de la vivienda y de los recursos económicos, cognitivos, sociales y políticos de los hogares, en función de la nacionalidad, como ya se hiciera desde finales de los sesenta con el concepto de «housing 
classes» (Rex, 1967). De este modo, la obsolescencia de los barrios históricos hace que los inmigrantes de menor renta y mayor fragilidad legal terminen habitando en estas zonas (Peach, 1975). En sentido opuesto, la potenciación del mercado de viviendas secundarias en ciertas áreas propicia, en un contexto de creciente globalización, la concentración de extranjeros de alto poder adquisitivo en ellas. Una cuarta línea de análisis se ha centrado en valorar la incidencia de las políticas de vivienda (Kesteloot, 1986) y del estado del bienestar en la distribución territorial de la población extranjera, factor que se ha argumentado para explicar la creación de guetos. Por último, para explicar la diferenciación espacial por nacionalidad no hay que olvidar los análisis que la relacionan con los ciclos del proceso migratorio.

Los más recientes enfoques en el análisis de la segregación se han centrado en la paradoja y cuestionada relación entre globalización y segregación (Wacquant, 2001; Kempen, 2007).

\section{La metodología}

\subsection{Las fuentes y sus deficiencias}

El estudio de la población extranjera que afrontamos en este artículo es un análisis estructural, es decir, considera ciertos atributos demográficos en un momento dado, como si se tratara de una foto en la que los procesos evolutivos han dejado una huella, aunque no puedan reconocerse sino a partir de los signos externos de la imagen. Esta perspectiva nos obliga a caracterizar el comportamiento territorial de la población extranjera a partir de la población «stock», según los datos que nos ofrecen los procedimientos estadísticos más recientes, el Censo de Población y Viviendas de 2001 y la explotación completa del Padrón de Población de 2006.

Los Censos de Población y Viviendas son el conjunto de trabajos que han permitido efectuar un recuento exhaustivo de la población y de las viviendas existentes en todo el territorio nacional español, aproximadamente cada diez años. En la actualidad los lleva a cabo el Instituto Nacional de Estadística (INE). Se inauguraron en 1857 y el último de ellos, el más completo de toda la historia censal española, tiene como fecha de referencia el uno de noviembre de 2001.

En el Censo de Población se incluyen todas las personas que tienen fijada su residencia habitual en nuestro territorio. Las variables analizadas son sexo, edad, lugar de nacimiento, nacionalidad, analfabetismo, estado civil, estudios terminados y en curso, lugar de estudio, migraciones, relación con la actividad económica, ocupación y paro, sector de actividad, situación profesional, 
lugar/horas de trabajo, medios/tiempo de desplazamiento al trabajo o estudio. Las cifras de población tienen una consideración puramente estadística, es decir, no son cifras oficiales de población.

Por su parte, el Padrón Municipal es el registro administrativo donde constan los vecinos del municipio. Sus datos constituyen prueba de residencia en dicho municipio y del domicilio habitual en el mismo. Su formación, mantenimiento, revisión y custodia corresponde a los ayuntamientos, de acuerdo con las normas aprobadas conjuntamente por el Ministerio de Economía y Hacienda y el Ministerio para las Administraciones Públicas, a propuesta del Consejo de Empadronamiento, obteniéndose la Revisión del Padrón Municipal con referencia a uno de enero de cada año.

En aplicación de la ley 4/1996, los ayuntamientos deben remitir, por medios informáticos o telemáticos, las variaciones mensuales que se vayan produciendo en los datos de sus padrones municipales al Instituto Nacional de Estadística, para que éste, en cumplimiento de sus obligaciones, realice las comprobaciones oportunas para subsanar posibles errores y duplicidades. Efectuadas dichas comprobaciones, el Instituto Nacional de Estadística obtiene una cifra de población para cada municipio, que eleva al Gobierno, quien la declara oficial mediante real decreto.

El Instituto Nacional de Estadística, a partir de estos datos, genera los ficheros que se utilizan para llevar a cabo la explotación estadística exhaustiva y que se pueden consultar en línea en la página web del Instituto Nacional de Estadística.

La utilización de estas fuentes administrativas no debe hacernos olvidar algunos de los problemas que presentan, en particular en lo que respecta a la población extranjera. Se trata de problemas derivados de su cobertura y fiabilidad, de los criterios de clasificación establecidos y de los cambios legislativos que han afectado directa o indirectamente al recuento de personas de nacionalidad extranjera o a la propia condición de extranjería.

Es más frecuente que se produzca una cierta subinscripción en el colectivo de población extranjera que en el del conjunto de la población, a consecuencia de la ausencia voluntaria de registro. Ello se debe a la situación de irregularidad de algunos inmigrados, a razones de multirresidencialidad, en el caso de los extranjeros que se asientan en Canarias por razones de ocio, y a las situaciones de desajuste que se dan con los procesos de nacionalización o que se derivan del tiempo que media entre la llegada de un extranjero y su inscripción. Por último, hay que considerar que la mayor parte de las bajas de extranjeros que se trasladan fuera de España no deja huella administrativa, lo que puede originar una sobredimensión de algunos colectivos. Por ello, hemos 
escogido también los datos del año 2006, pues los ayuntamientos, de acuerdo con la actual normativa, practicaron ese año, por primera vez, las oportunas correcciones, dando de baja a los residentes extranjeros no comunitarios que no habían renovado el permiso de residencia en los dos años anteriores.

\subsection{El microanálisis y los datos geodemográficos}

En la actualidad, desde una perspectiva geográfica, se hace necesario trabajar con un gran nivel de detalle estadístico si se quiere atender a la riqueza que representa la amplia gama de escalas que permiten explicar los procesos que afectan al territorio, en correspondencia con las distintas situaciones demográficas que se viven en ámbitos geográficos segregados socialmente (Aldrey, 2005; 509). Se trata, por tanto, de reconocer las homogeneidades o discontinuidades que se pueden advertir en función de las características de la población residente en los distintos ámbitos administrativos, lo que vuelve definitivo el tamaño de la unidad de análisis con que componemos y descomponemos el área de estudio (los municipios, los distritos, las secciones...).

El microanálisis sociodemográfico tiene como objeto las agregaciones de población asociadas a una reducida base espacial, un enfoque que tiene un gran interés, puesto que los procesos por los que se fragmenta el territorio en áreas sociales diferenciadas constituyen una de las evidencias más genuinas de la naturaleza social del espacio. En consecuencia, en el análisis microescalar que aquí desarrollamos se asumen, como unidades de observación, las agregaciones de población correspondientes a pequeñas demarcaciones espaciales, que se adoptan como las piezas menores a partir de las cuales se pueden determinar los comportamientos territoriales (Ocaña, 2005).

Desde el punto de vista de las fuentes demográficas, dejando al margen las restricciones que impone el secreto estadístico, conviene reconocer que la información digitalizada que se ha ido consolidando en los últimos años en España, a iniciativa del Instituto Nacional de Estadística y de algunos otros organismos competentes en esta materia en las distintas comunidades autónomas, ha abierto posibilidades antes desconocidas para el microanálisis espacial. Por primera vez en la historia estadística española, el Censo de 2001 ofrece una cuantiosa información inframunicipal (entidades de población, núcleos de población, distritos y secciones) para todo el territorio nacional. No conviene olvidar que hasta el año 1981 la información de muchos de los censos no cubría siquiera a todos los municipios. Por tanto, la senda que abre el nuevo tratamiento del Censo, con esta perspectiva escalar, tiene un valor extraordinario para geógrafos e investigadores sociales. 
En cuanto a la explotación estadística del Padrón, desde 1996, el sistema de gestión padronal comenzó a ofrecer datos anuales a partir de las revisiones que se realizan con referencia a uno de enero de cada año y que derivan de una actualización continua. Aunque el número de variables que tienen aprovechamiento estadístico (sexo, edad, nacionalidad, lugar de nacimiento, lugar de residencia, y relación entre lugar de residencia y lugar de nacimiento) es mucho menor que el de las que aporta el Censo, el Padrón ofrece la enorme ventaja de disponer de una información actualizada. Además, con su explotación, el INE inauguró la producción de datos por secciones censales. Concretamente ofreció ocho tablas con referencias al sexo, la edad y el origen de la población para los años de 1998 a 2003. A partir de 2004 se pueden consultar en línea. En el año 2006, además, se ha incrementado la explotación porque se ha sumado la relación entre lugar de nacimiento y residencia a los datos que ya se suministraban de población por sexo, edad y nacionalidad.

Por último, completan este abanico de características las posibilidades de descargar los ficheros en formato de hojas de cálculo (Excel) que, a su vez, pueden ser manipuladas para vincularlas a otros programas de cálculo más complejos (SPSS) y a los Sistemas de Información Geográfica.

\subsection{La elaboración del Sistema de Información Geodemográfica}

Un Sistema de Información Geográfica como integración organizada de hardware, software y datos geográficos y personales, está diseñado para capturar, almacenar, manipular, analizar y desplegar en todas sus formas la información geográficamente referenciada. En este caso la que vinculamos al Sistema es de carácter demográfico y por ello lo hemos definido como Sistema de Información Geodemográfica.

El modelo de Sistema de Información Geográfica que hemos utilizado es el de un SIG vectorial, dado que los fenómenos que se representan son discretos, es decir, de límites definidos. En el caso que nos ocupa se trata de las unidades administrativas (secciones censales, distritos censales y municipios) que tienen unos contornos precisos y que, por tanto, se pueden gestionar mediante una topología de polígonos. Hemos georrefenciado a esta topología una serie de datos que recogen las variables demográficas que hemos utilizado para nuestro análisis.

La construcción del Sistema de Información Geodemográfica requirió de la utilización de la cartografía digitalizada de los distintos municipios, distritos y secciones censales del Instituto Canario de Estadística (ISTAC), así como de los datos geodemográficos del Padrón, que hubo que vincular a ella. Sin embargo, cuando afrontamos dicha tarea nos encontramos ante un desfase 
temporal ya que la cartografía había sido elaborada el año 2002 y los datos de referencia eran de 2006. Por tanto, cuando contrastamos la identidad de las parcelas correspondientes a las secciones censales con las etiquetas o códigos que aparecían en la información padronal había ciertas diferencias que hubimos de resolver. En algún caso fue necesario agregar los datos de dos secciones censales, puesto que la subdivisión no sólo requería digitalizar nuevos límites para poder subdividir una antigua sección censal en dos, sino también saber exactamente el trazado de esos nuevos límites. Otras veces hubo que sumar parcelas para atender a una nueva clasificación agregada y, en ambas circunstancias, tuvimos que consultar con los ayuntamientos afectados la distribución territorial para ajustarla convenientemente a la información demográfica.

Otra de las actividades que afrontamos en la construcción del Sistema de Información Geodemográfica fue la de conferir un nuevo atributo de texto a la denominación de las secciones censales. Se nos hacía muy complejo trabajar con códigos numéricos cuando, tras ese código, latía una realidad territorial cuyo reconocimiento exigía de nuestra aprehensión geográfica. Por ese motivo, combinamos la información de las secciones censales con la de las entidades de población y asignamos a cada sección censal el nombre de la entidad más populosa que contenía. Esta laboriosa tarea se ha visto recompensada cuando nos hemos enfrentado a los distintos mapas y a su interpretación.

\subsection{La explotación del Sistema de Información Geodemográfica}

El punto de partida de nuestro análisis es medir la presencia de extranjeros en cada una de las secciones censales y explicar la distribución territorial de la extranjería en las islas. Con este fin se utilizó, en cada unidad de análisis, el índice de extranjería, entendiendo como tal la ratio del número de extranjeros por cada cien españoles. Este primer indicador permitió reconocer numérica y cartográficamente dónde se encontraban empadronados en 2001 y 2006 los extranjeros residentes en Canarias. Los factores que determinan su distribución también fueron enunciados y cartografiados pero, en este caso, las dificultades que teníamos para obtener la información desagregada a nivel de sección nos obligaron a utilizar el municipio como unidad espacial de referencia.

El análisis de la distribución del porcentaje de extranjeros por sección revela un comportamiento muy alejado de la normalidad estadística. Esto implica que el valor del índice es muy reducido en la mayor parte de las secciones, frente a otras pocas en las que se alcanzan valores muy elevados. Es cierto que esta heterogeneidad viene dada por la propia distribución territorial del fenómeno pero es también una consecuencia del análisis a microescala. 
La peculiar distribución de la variable puede inducir a que determinados análisis estadísticos, como el estudio de las tendencias de crecimiento, el establecimiento de coeficientes de localización o el cálculo de índices de segregación, generen resultados alejados de la realidad. En otras palabras, el escaso número de extranjeros en algunas unidades espaciales puede repercutir en la generación de tendencias y relaciones que carecen de significación estadística, motivadas por el azar o magnificadas falazmente.

Era necesario, por tanto, utilizar unidades espaciales más homogéneas, con el fin de que cualquier análisis admita un enfoque comparativo. Por este motivo, se realizaron dos selecciones espaciales, una para cada momento estadístico, que incluyen las secciones en las que la extranjería alcanza un umbral mínimo. A estas selecciones las hemos llamado «espacios de la inmigración» en Canarias, en contraposición al resto del territorio de las islas, que podría ser definido como «espacios de repulsión».

La selección de las secciones se realizó a partir de los índices de extranjería. Dado que los índices promedios para el conjunto de las secciones censales eran del $5,2 \%$ y $9,2 \%$ en 2001 y 2006 , respectivamente, y que las dispersiones respectivas eran elevadas $(7,2$ y 11,2), se decidió determinar un umbral mínimo de selección, que se estableció a partir de la resta del valor de media dispersión típica respecto al promedio de la variable. De este modo, las secciones que forman parte de los espacios de la inmigración contaban con una población extranjera que suponía, al menos, el 2\% de su población en 2001 y el 4\% en 2006, lo que, a su vez, implicaba que el contingente mínimo en términos absolutos fuera de 84 extranjeros para el primer año y 184 para el segundo.

Como consecuencia, la muestra espacial incluyó en torno al 25\% de las secciones (295 de las 1179 secciones existentes en 2001 y 296 de las 1204 de 2006). El $83 \%$ de las secciones coinciden en ambas selecciones espaciales. El resto, unas 40 secciones, no aparecen en ambas muestras, bien porque se ha reducido la presencia de extranjeros, en términos relativos, en el lustro que separa a ambos momentos censales, bien porque se producen cambios en la cartografía territorial de las secciones del archipiélago, a través de la segregación o agregación de unidades espaciales.

El segundo objetivo del análisis se centra en reconocer las diferencias en la localización según nacionalidad, en el establecimiento de modelos territoriales diferenciados para cada una y en la determinación de los factores de localización incidentes. Para lo primero se hallaron los coeficientes de localización de las 19 nacionalidades con mayores contingentes en 2006, para las cuatro islas donde se concentra la mayor parte de los extranjeros (Fuerteventura, 
Gran Canaria, Lanzarote y Tenerife). Este coeficiente ${ }^{2}$ relaciona la población de una determinada nacionalidad en una sección concreta, respecto a la población total de esa sección, y respecto a la población total y por nacionalidad de los territorios de la inmigración de la isla. El resultado del coeficiente permitió reconocer la distribución territorial de cada una de las nacionalidades. Algunas de las más representativas (alemanes, británicos, colombianos y marroquíes) aparecen cartografiadas. Además, el coeficiente permitió establecer la existencia de modelos espaciales concentrados o dispersos, para lo cual se utilizó como indicador sintético el índice de variación por nacionalidad, que se obtiene relacionando el valor promedio del coeficiente con la dispersión estándar de la variable.

Los diferentes patrones de distribución y los diversos modelos espaciales de concentración permitieron definir al menos dos perfiles migratorios y establecer varios factores de localización. El agrupamiento de las nacionalidades por perfiles se hizo mediante correlaciones bivariadas.

El tercer objetivo del trabajo es la medición de los niveles de segregación geográfica por nacionalidad de la población extranjera residente en Canarias. Existe una gran variedad de indicadores cuantitativos dirigidos a medir las cincos dimensiones que tradicionalmente se han señalado como características de la segregación geográfica: igualdad, exposición, concentración, centralidad y agrupamiento (Massey y Denton, 1988). La selección de un determinado indicador depende de la naturaleza de los datos, de las características de las unidades de análisis, de la escala (Sabatini et al., 2001) y del objetivo del índice. Éramos conscientes de que gran parte de los estudios se basan en el índice D de disimilitud (Duncan y Duncan, 1955) y de que éste tiene una gran potencialidad cuando es utilizado en un sistema de información geográfica, dado que ha sido mejorado notablemente con posteriores revisiones que han introducido fundamentos de estadística espacial en su cálculo (Morrill, 1991; Wong, 1993 y 2005). Sin embargo, teniendo en cuenta que abordábamos en nuestro estudio la segregación a escala insular, se consideraban unidades espaciales relativamente pequeñas que no se localizaban de manera continua en el espacio. Por este motivo, aplicamos el índice sintético de segregación a las 19 nacionalidades con mayores contingentes de Canarias. El índice relaciona la población total y por nacionalidad de la sección con la población total y

2. $Q L=\frac{(X i / T i)}{(X / T)}$, donde $\mathrm{X}_{\mathrm{i}}$ es la población por nacionalidad de una sección determinada, $T_{i}$ es la población total de la sección, $X$ es la población por nacionalidad en los territorios de la inmigración de cada isla y $\mathrm{T}$ es la población total en los territorios de la inmigración de cada isla. 
por nacionalidad de la isla y permite valorar en términos comparativos si las nacionalidades se distribuyen de un modo segregado en el espacio ${ }^{3}$.

Por último, el Sistema de Información Geográfica permite abordar enfoques desde una perspectiva estrictamente territorial. En este sentido, nos propusimos como cuarto objetivo caracterizar el grado de especialización espacial de cada una de las secciones incluidas en el análisis, en función de la nacionalidad de los extranjeros empadronados en ellas. Teniendo en cuenta que habíamos definido dos perfiles migratorios fundamentales en las islas, se midió el grado de especialización de las diferentes secciones mediante el coeficiente de localización, que compara el número de extranjeros de un perfil, en una sección determinada, con el total de extranjeros empadronados en esa sección, el total de extranjeros en la isla y el total de extranjeros de ese determinado perfil en la isla. Como resultado definimos cuatro categorías distintas, dos alusivas a una clara especialización en algunos de los perfiles y las otras dos relativas a una situación de ligera especialización. El resultado final permitió una cartografía ilustrativa.

\section{La extranjería en Canarias}

A lo largo de la historia, la posición geográfica del archipiélago y las peculiaridades de su economía agraria y de su especialización turística le han conferido un papel de encrucijada entre los continentes europeo, africano y americano, por lo que la extranjería no se ha de considerar un fenómeno nuevo en Canarias. Sin embargo, en los últimos años, la inmigración exterior que había venido evolucionado al compás de los vaivenes del desarrollo económico, ha adquirido una nueva dimensión. A la llegada de extranjeros de Europa occidental, por motivaciones residenciales o laborales, vinculadas a la actividad turística, y de otros provenientes de orígenes antaño más exóticos (palestinos, hindúes, coreanos, etc.), se ha sumado el retorno de antiguos emigrantes de naciones latinoamericanas, así como una inmigración generalizada de todo origen y condición, propia de la globalización de las migraciones humanas.

Desde una perspectiva cronológica, el desarrollo turístico que tuvo lugar desde la década de los años sesenta del siglo xx determinó una modificación del balance migratorio en Canarias, que pasó a ser positivo tras un largo período de predominio de la emigración. El fortalecimiento de la especialización

\footnotetext{
3. $I S=\frac{1}{2} \sum_{i=1}^{n}\left|\frac{x_{i}}{X}-\frac{t_{i}-x_{i}}{T-X}\right|$, donde $x_{i}$ es la población por nacionalidad en las secciones, $\mathrm{X}$ es la población por nacionalidad en el municipio, $\mathrm{t}_{\mathrm{i}}$ es la población total de la sección y $\mathrm{T}$ es la población total del municipio.
} 
turística del archipiélago, así como la nueva situación sociopolítica española y los avances económicos de los años setenta y ochenta, influyeron en el atractivo de Canarias para ciudadanos extranjeros de distintas procedencias. Este atractivo se vio reforzado, desde 1986, con la incorporación de España a la Unión Europea y el proceso de convergencia con otros países más desarrollados de su entorno. Por último, desde fines del siglo XX, a estas circunstancias se debe sumar el impacto de los procesos de globalización, que han contribuido de forma decisiva al incremento de las corrientes de inmigración.

Según datos del Censo, en 1991 residían en Canarias unas 32000 personas de nacionalidad extranjera, lo que representaba alrededor del 2,1\% de la población. En esa misma fecha, en España, tan sólo el 0,91\% de los residentes era extranjero. Sin embargo, a medida que avanza la última década del siglo XX y la primera del siglo XXI, el ritmo de establecimiento de extranjeros se acelera. El avance de la explotación estadística del Padrón de 2007 informa de un $12,31 \%$ de extranjeros en Canarias ( 248871 personas), a fecha uno de enero de ese año, al mismo tiempo que en España se ha alcanzado la cifra de 9,93\% (4482568 personas). Todo esto refleja una situación excepcional en el contexto de los países de la Unión Europea, debido a que la inmigración ha tenido un crecimiento muy concentrado en el tiempo en el tránsito de los siglos XX al XXI, tanto en Canarias como en el conjunto de España, como pone de manifiesto la recta de regresión que se registra para estos últimos nueve años (figura 1).

De forma complementaria a estos datos, debemos tener en cuenta que otra de las características más sobresalientes de la inmigración que ha tenido lugar en estos últimos años es la de la diversificación de procedencias. Así, por ejemplo, en el año 2007 había ciudadanos de unos 200 países distintos residiendo en Canarias, lo que pone de manifiesto la globalización de las migraciones, es decir, la internacionalización de la economía y la activación de nuevos flujos migratorios.

Desde el punto de vista de las distintas nacionalidades, el flujo de población extranjera que predominó en la etapa comprendida entre 1975 y 1990 provenía de Europa Occidental y, aunque algunos inmigrados de esta procedencia trabajaban en ciertas actividades del sector turístico, era muy superior la inmigración no vinculada a razones laborales, sino a motivaciones turísticoresidenciales. No obstante, entre las procedencias no europeas, la inmigración laboral tuvo un papel complementario en el seno de la estructura productiva de Canarias, en el sector del comercio (hindúes, marroquíes), en el de la pesca (rusos, ucranianos, japoneses y coreanos), en el servicio doméstico (filipinos) y en las actividades vinculadas al sector turístico (británicos, alemanes, etc.). 


\section{Figura 1}

Recta de regresión de los extranjeros residentes en Canarias

$y=23839 x-5 E+07$

$R^{2}=0,9806$

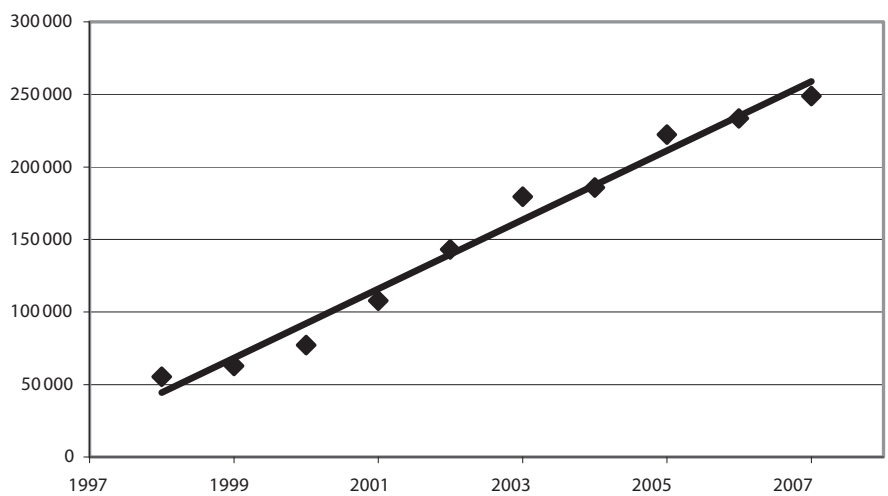

Fuente: Padrón continuo de población. INE. Elaboración propia.

En los últimos años, a la fuerte presencia de residentes europeos comunitarios, el colectivo de extranjeros de mayor importancia numérica en Canarias, se suma el protagonismo que ha adquirido la inmigración de procedencia latinoamericana. En algunos casos se trata de emigrantes retornados o de sus descendientes, en otros, de personas que, gracias a las privilegiadas relaciones que España mantiene con América Latina, han acudido atraídos por un bienestar económico que contrasta con las situaciones de crisis vividas por algunos de aquellos países. Ésta es la interpretación que cabe dar al hecho de que, en 2007, tras los primeros colectivos de residentes extranjeros, alemanes (35574), británicos (33705) e italianos ${ }^{4}$ (20714), los colombianos constituyan el cuarto contingente de inmigrantes que reside en Canarias (19314) y los venezolanos el sexto (11065). Con la excepción de los marroquíes, que se sitúan en quinta posición y que tienen una dimensión que les otorga el primer puesto de entre las nacionalidades africanas (14059), se hallan otros dos contingentes de origen latinoamericano, con cifras próximas a los 10000 residentes cada uno, argentinos y cubanos.

En un análisis detallado de las nacionalidades, si seguimos la evolución de los datos padronales desde 1998 a 2007, se pone de manifiesto la

4. Algunos de los residentes italianos que viven en Canarias nacieron en Argentina o Uruguay, pero han recuperado la nacionalidad de sus antepasados de origen italiano. 
Figura 2

Ejemplo de crecimiento lineal y constante (Bolivia)

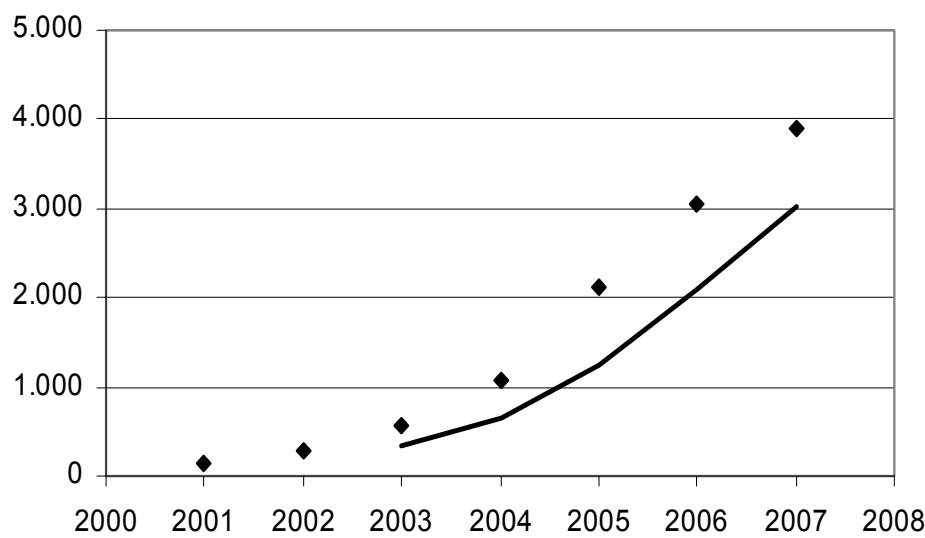

Fuente: Padrón continuo de población. INE. Elaboración propia.

diversificación reciente de la inmigración, hasta tal punto que muchos de los contingentes de mayor peso demográfico han reducido su participación (Alemania o India, por ejemplo) mientras que otros de menor significación la han reforzado notablemente (Bolivia, China o Uruguay, por ejemplo). Esta tendencia a la diversificación queda parcialmente distorsionada por el hecho de que desde la rectificación padronal de 2006, cuando se dio de baja a los extranjeros no comunitarios que no habían renovado el permiso de residencia, no se hizo lo mismo para con los ciudadanos comunitarios, de tal forma que las cifras de los europeos comunitarios pueden estar ligeramente sobredimensionadas.

En cualquier caso, las diferencias en las tendencias de crecimiento de las distintas nacionalidades permiten apreciar la diversificación de corrientes de inmigración. De esta manera, la evolución demográfica medida a través de medias móviles, en el caso de ciertos colectivos de escaso peso demográfico, ofrece una trayectoria de crecimiento claramente lineal, como sucede con Bolivia (figura 2).

Otras nacionalidades más representadas, sin embargo, como los alemanes o argentinos, manifiestan un crecimiento más atenuado, porque se han visto afectadas por ligeros retrocesos (figuras 3 y 4 ). 


\section{Figura 3}

Ejemplo de crecimiento con ligero retroceso y recuperación (Alemania)

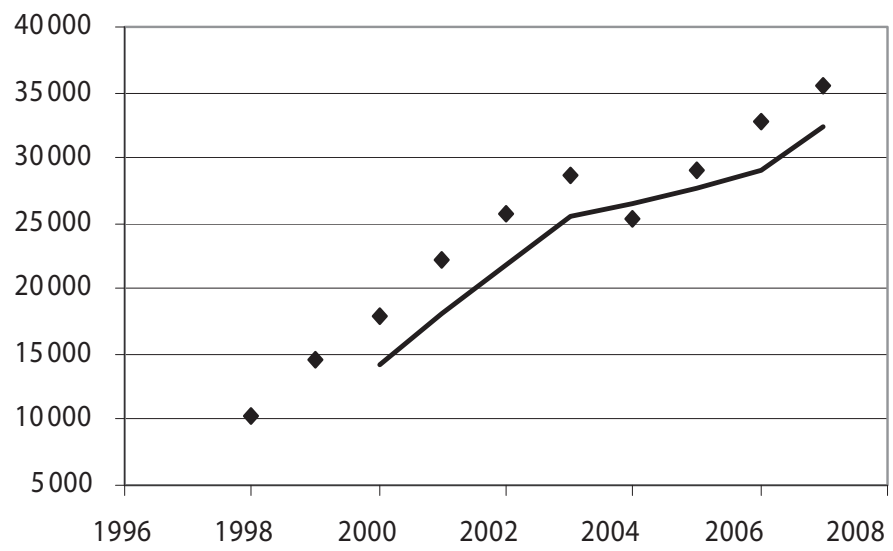

Fuente: Padrón continuo de población. INE. Elaboración propia.

Figura 4

Ejemplo de crecimiento inicial y posterior estancamiento (Argentina)

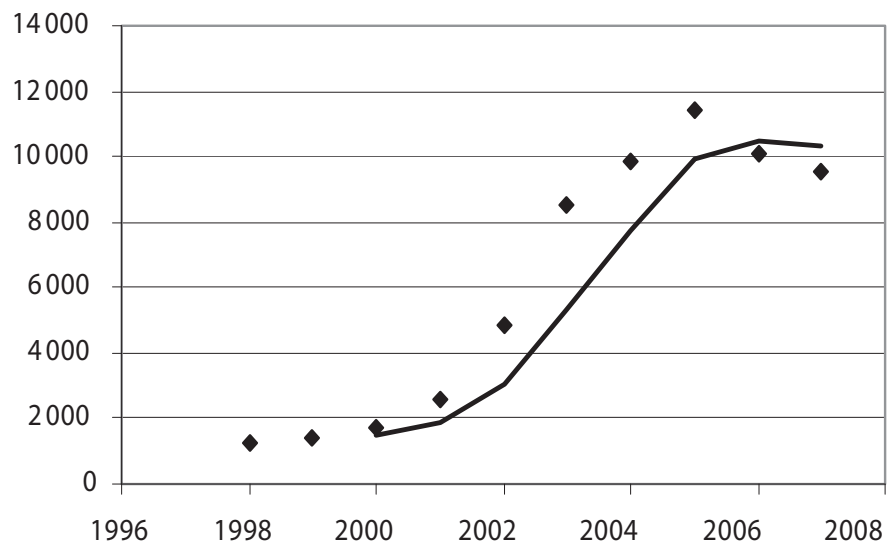

Fuente: Padrón continuo de población. INE. Elaboración propia.

\section{Pautas de distribución territorial de la población extranjera}

El análisis de la localización espacial de la población extranjera se ha realizado a partir del índice de extranjería (ratio del número de extranjeros por cada cien españoles) que se registra en cada sección. En 2006, el índice promedio 
Figura 5

Índices de extranjería en 2006 por sección

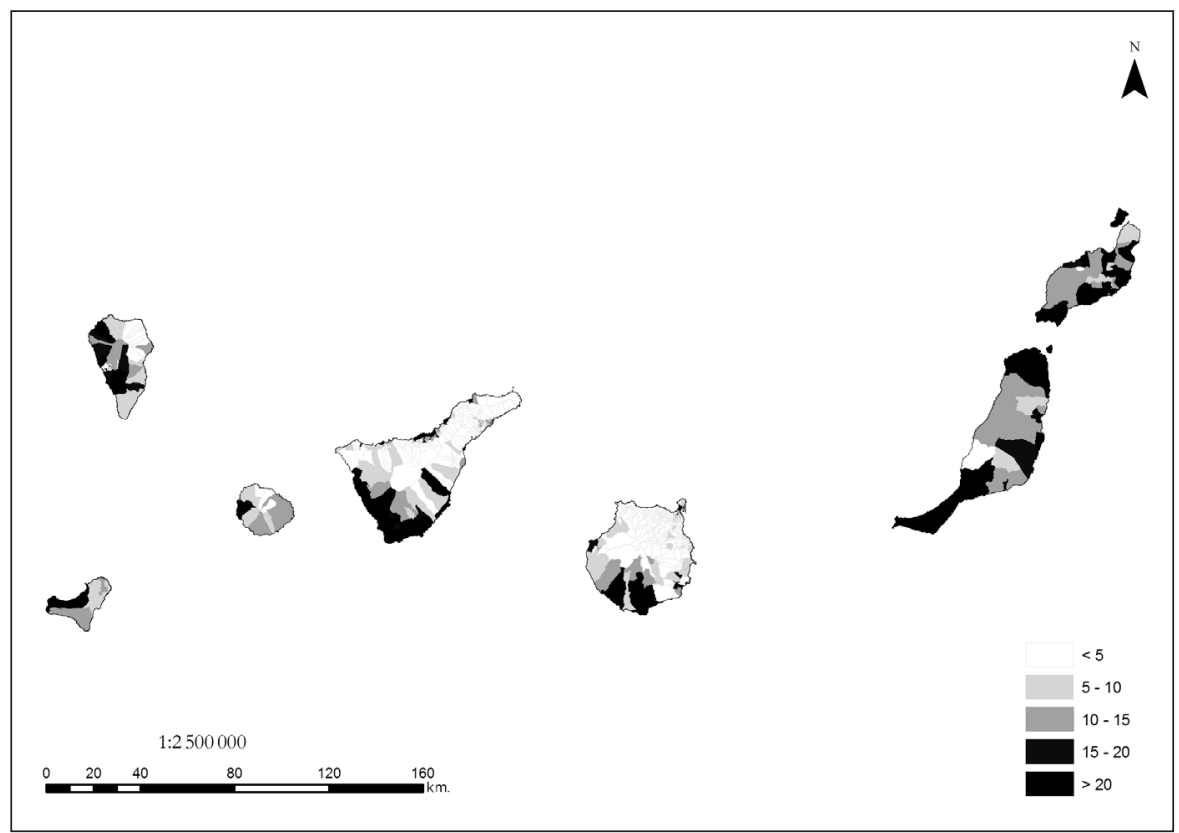

Fuente: Padrón continuo de población. INE. Elaboración propia.

para el conjunto de las 1204 secciones era de 12,8 pero se registraba una enorme heterogeneidad, como lo atestigua el que la desviación estándar fuera de 23,2 y que la distancia entre el valor mínimo y el máximo fuera de 254,7 puntos, es decir, que había secciones censales de índice cero, mientras que en otras estaban empadronados 2,5 extranjeros por cada español. Esta amplitud denotaba una considerable diferencia entre las distintas unidades de análisis territorial.

En realidad, una gran parte del territorio no registraba una significativa presencia de población extranjera o, lo que es lo mismo, una amplia superficie de la geografía insular era un área de repulsión para el asentamiento de esta población. De hecho, la mitad de las secciones registraba índices inferiores a cinco, por debajo del valor mediano de la distribución de la variable $(5,3)$.

Desde una lectura inversa, la inmigración tenía una enorme repercusión en un ámbito territorial cuyas dimensiones se circunscribían, principalmente, a las áreas de mayor especialización turística: Valle Gran Rey en La Gomera, Puerto de la Cruz en el norte de Tenerife, costas del sur-suroeste de esta 


\section{Figura 6}

Plazas turísticas/municipios. 2006

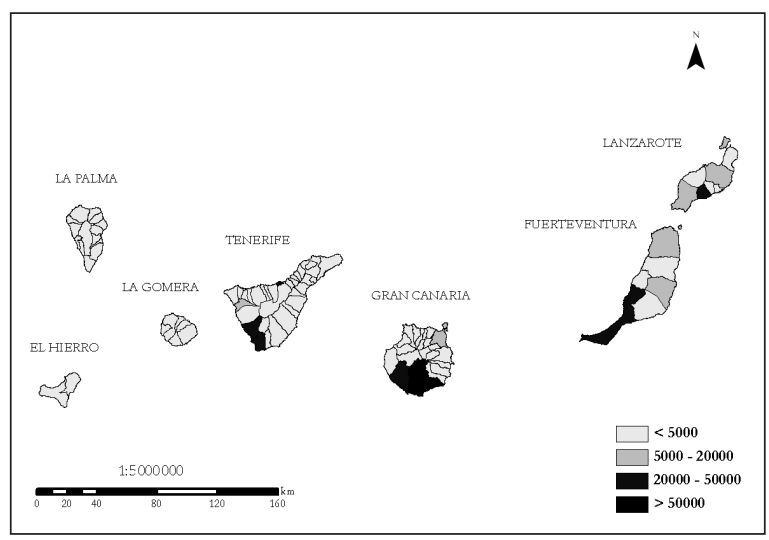

Figura 7

Viviendas secundarias/municipios. 2001

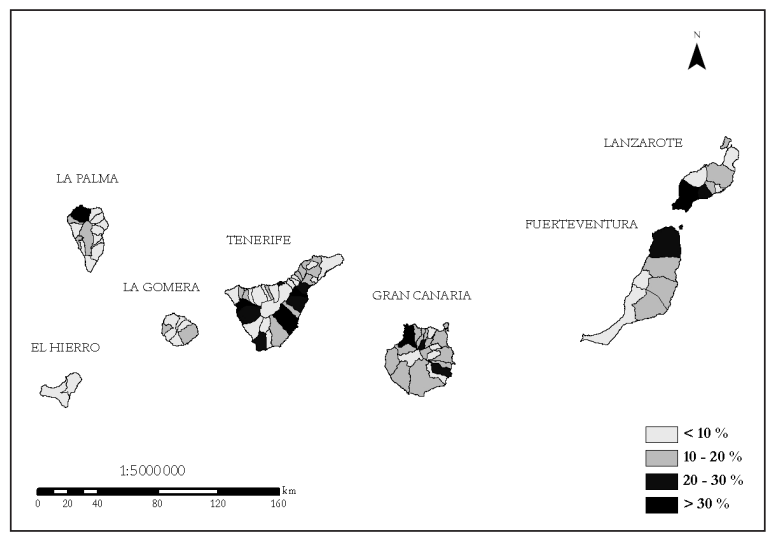

Fuente: Padrón continuo de población. INE. Elaboración propia.

misma isla, costas del sur-suroeste de Gran Canaria y un número elevado de secciones donde se han edificado las urbanizaciones turísticas y residenciales de Lanzarote y Fuerteventura. En consecuencia, los mapas de las figuras 6 y $7^{5}$ ponen de manifiesto que las funciones turística y residencial juegan un papel

5. El mapa se refiere al año 2001 pues se ha elaborado con la información del Censo de Población y Viviendas de 2001, la única fuente de que disponemos para analizar este hecho. 
primordial en la presencia de población extranjera. Se trata de los espacios que acogen los complejos turísticos o las viviendas rurales para población retirada, en entornos de elevado valor paisajístico.

No obstante, un análisis más detallado nos obliga a considerar otras causas de orden secundario. En primer lugar, los procesos de urbanización y de las funciones a ellos asociadas (comerciales, portuarias, servicios personales), que explican que se registren importantes índices de extranjería en las capitales de Canarias, las ciudades de Las Palmas de Gran Canaria y de Santa Cruz de Tenerife, o en las periferias residenciales de las áreas turísticas. Se trata de ámbitos que, tradicionalmente, venían acogiendo a ciertas comunidades de inmigrados y que hoy siguen ejerciendo una importante atracción para la población extranjera que escoge un destino urbano (figura 8).

En segundo lugar, no hay que olvidar las cuotas de empleo de mano de obra extranjera en la agricultura de exportación, una actividad que al ser repudiada por la población autóctona ha representado un importante nicho de empleo para ciertas comunidades de inmigrados. Algunos municipios especializados en la agricultura intensiva de productos extratempranos registran elevados índices de población extranjera por esta razón (figura 9). Por último, la llegada de latinoamericanos y caribeños, hijos o nietos de antiguos emigrantes canarios, permite explicar que el índice de extranjería sea considerable en ciertos ámbitos urbano-turísticos que han acogido también a los retornados ${ }^{6}$ (figura 10).

En consecuencia, el modelo territorial de la extranjería en Canarias está estrechamente vinculado al binomio turismo-actividades urbanas. Sin embargo, una comparación entre los datos censales de 2001 y los padronales de 2006 pone de manifiesto que, en la primera fecha de referencia, la influencia de aquella especialización económica era mayor, de forma que las secciones con mayores tasas de extranjería se circunscribían aún más, geográficamente, a los espacios urbanos y turísticos.

Desde nuestro punto de vista esto indica que se han diversificado los factores de atracción residencial, por el creciente número de actividades que conforman un mercado laboral de carácter global y por el proceso de articulación e integración de los espacios económico-residenciales a escala insular, que se ha reforzado durante este período. En relación con este último factor, la

6. Como no disponemos de información fidedigna de retornados, hemos utilizado la información de los venezolanos que en el año 2005 se dieron de alta como residentes en los municipios de Canarias, que alcanzaron la cifra de 3601 en ese año. Conviene recordar que Venezuela fue el principal destino de la emigración de Canarias en los años cincuenta, sesenta y setenta del siglo XX. 
Figura 8

Población/municipios. 2006

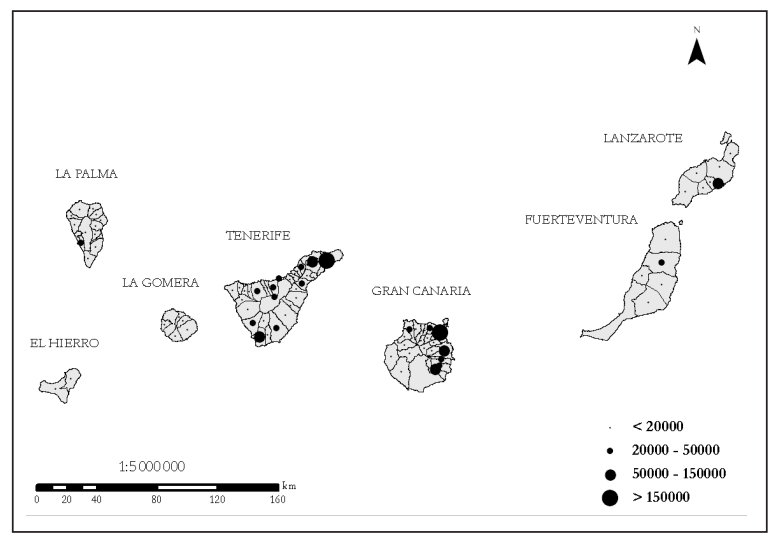

Figura 9

Empleo agrario/municipios. 2006

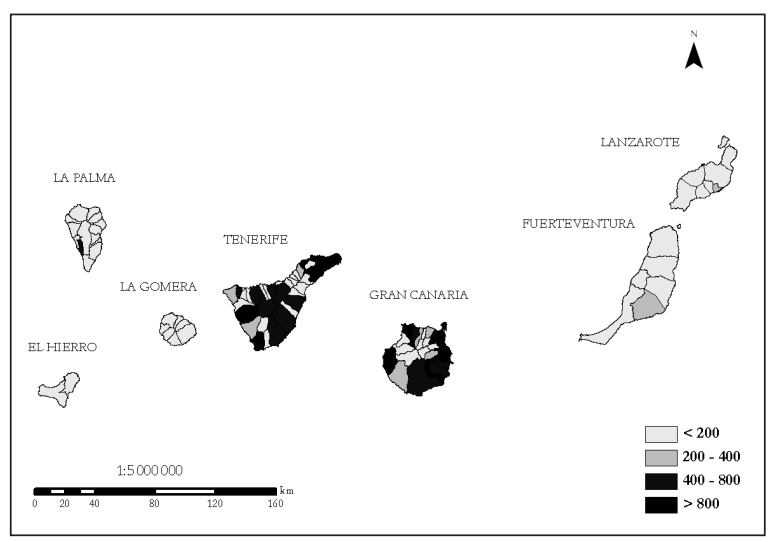

Fuente: Padrón continuo de población. INE. Elaboración propia.

relación migración-integración económica podría ser de carácter bidireccional, ya que a las mejoras en las vías de comunicación, que favorecen la movilidad por razones económico-residenciales, se ha sumado el menor arraigo de los nuevos residentes al territorio de acogida. Todo ello ha contribuido a incrementar los flujos intrainsulares.

El aumento de los colectivos extranjeros (crecimiento), la diversificación de las motivaciones por las que residen en Canarias (globalización) y el incremento de la articulación territorial de los espacios insulares (integración) 
Figura 10

Altas de ciudadanos venezolanos en municipios de Canarias en 2005

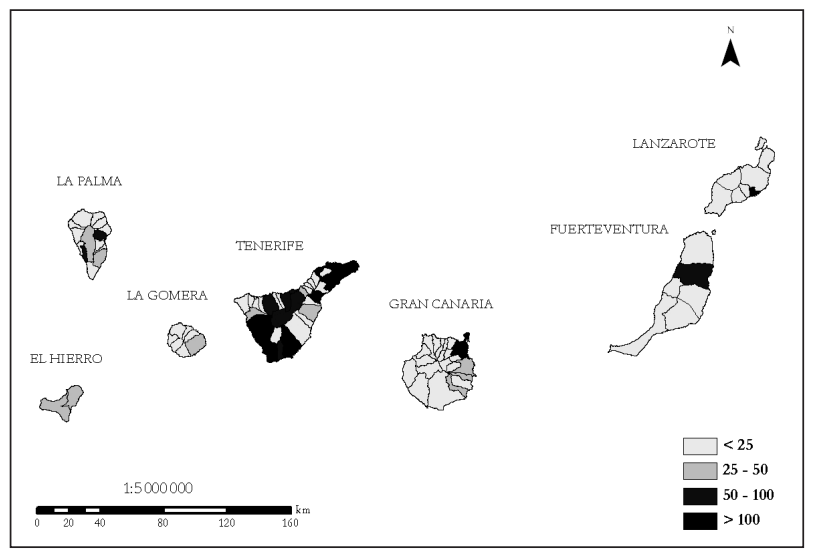

Fuente: Padrón continuo de población. INE. Elaboración propia.

están motivando que el modelo territorial de la extranjería haya evolucionado hacia una mayor homogeneidad (el índice de variación ha descendido de 1,9 a 1,8 en tan sólo cinco años). No obstante, la actividad turística sigue condicionando los flujos migratorios de foráneos. Esto queda de manifiesto si comparamos los índices de extranjería registrados para los territorios de la inmigración en 2001 y 2006. La diferencia entre estos índices muestra que, salvo en cuatro secciones, se produce una tendencia general al crecimiento de la población extranjera (incremento medio de 19,8 puntos) y que aquellas que registran un mayor aumento (superior a 40 puntos) son secciones en las que se concentra la oferta turística. Se trata de unidades en las que se ha desarrollado un tejido hotelero y extrahotelero de reciente creación, como sucede en algunos de los municipios de Yaiza o Tías en Lanzarote, de Arona y Adeje en el suroeste de Tenerife o de Mogán en el suroeste de Gran Canaria, en las que los crecimientos oscilan entre los 60 y los 139 puntos. En cambio, las secciones de las capitales insulares, las de las áreas residenciales cercanas a los espacios turísticos y las de otros espacios residenciales de naturaleza «rururbana», registran incrementos, como mucho, de entre 20 y 40 puntos.

\section{El comportamiento espacial por nacionalidades}

El análisis de la distribución de la población extranjera en Canarias por secciones ha puesto de manifiesto la relevancia del fenómeno turístico. Cabe 
ahora preguntarse si existen patrones territoriales y otros factores de incidencia además de éste en función de la nacionalidad. Para ello pretendemos analizar el comportamiento territorial de las nacionalidades con un mayor peso en las islas por secciones censales (con el fin de obtener totales absolutos representativos), utilizando un coeficiente de localización que relacione la población de una determinada nacionalidad, en una sección concreta, respecto a la población total de esa sección y a la población total de los territorios de la inmigración de la isla, de tal modo que se pueda apreciar si dichos residentes presentan un comportamiento disperso o concentrado, qué pautas de localización siguen e inferir los factores de localización incidentes. Para ello nos vamos a centrar en las cuatro islas en las que el contingente de extranjeros permite obtener resultados significativos en esta escala de análisis: Tenerife, Gran Canaria, Lanzarote y Fuerteventura.

En primer lugar, los índices de variación de los coeficientes de localización obtenidos para las secciones censales consideradas en los espacios de la inmigración muestran la presencia de patrones territoriales diferentes en función de la nacionalidad. Italianos, portugueses, argentinos, colombianos, venezolanos y cubanos se distribuyen en el territorio de un modo disperso, frente al resto, que tiene un comportamiento concentrado. Destaca el elevado índice de variación registrado por los británicos, belgas, chinos, bolivianos y mauritanos en casi todas las islas y, con algunas excepciones, los registrados por alemanes, holandeses, franceses y rumanos, así como los ecuatorianos y marroquíes en Tenerife (véase el cuadro 1). La presencia de distintos modelos territoriales nos hace deducir la existencia de diferentes perfiles migratorios y la desigual incidencia de los factores de localización en función de la nacionalidad. Pero también nos permite deducir que los factores de localización difieren de una isla a otra.

El cálculo de correlaciones bivariadas por nacionalidad para las cuatro islas consideradas nos revela que podemos clasificar las nacionalidades en dos grandes grupos en función de su localización: el formado por las nacionalidades de la Unión Europea, que salvo algunas excepciones, mantienen coeficientes positivos entre ellas, y el resto de las nacionalidades. Esta separación refleja la presencia de dos tipologías migratorias diferentes, la primera de carácter turístico-laboral y, la segunda, estrictamente laboral.

Entre los europeos, por tanto, la localización la determina un tejido turístico-residencial vinculado con el mercado internacional del turismo de masas o, lo que es lo mismo, el factor que condiciona la presencia de los comunitarios es el del desarrollo de una actividad inmobiliaria destinada al mercado 
europeo en zonas litorales, con óptimas condiciones climáticas durante todo el año.

Ahora bien, si en los cuatro casos considerados el mercado inmobiliario es el principal condicionante ¿por qué se registran diferentes modelos territoriales según nacionalidad e isla? Las razones son de dos tipos, ya que existen otros factores que debemos considerar. En primer lugar, no podemos olvidar que se registra cierta inmigración europea de carácter laboral, que al vincularse no sólo con las actividades turísticas sino también con otras de carácter eminentemente urbano, tiene un comportamiento menos concentrado en el territorio. Este es el caso de los portugueses y de los italianos en todas las islas, que mantienen un patrón territorial disperso por la coalescencia de factores residenciales y laborales o el de los franceses presentes en Gran Canaria, que residen en un importante número en la capital insular (Las Palmas de Gran Canaria), al tratarse de extranjeros que trabajan en actividades terciarias no turísticas.

Cuadro 1. Índices de variación de los coeficientes de localización por islas y nacionalidad

\begin{tabular}{|l|c|c|c|c|}
\hline & Fuerteventura & Gran Canaria & Lanzarote & Tenerife \\
\hline Alemania & 1,5 & 1,9 & 1,6 & 1,1 \\
\hline Bélgica & 1,4 & 1,7 & 1,4 & 1,7 \\
\hline Francia & 1,3 & 1,1 & 1,4 & 1,6 \\
\hline Italia & 0,9 & 0,9 & 1,0 & 1,1 \\
\hline Países Bajos & 1,2 & 1,7 & 1,7 & 1,0 \\
\hline Portugal & 0,8 & 0,9 & 0,9 & 1,1 \\
\hline Reino Unido & 1,8 & 2,0 & 1,5 & 1,5 \\
\hline Rumanía & 1,8 & 1,8 & 1,1 & 1,4 \\
\hline Argentina & 0,7 & 0,8 & 0,7 & 0,8 \\
\hline Bolivia & 2,7 & 1,8 & 1,8 & 2,7 \\
\hline Colombia & 0,8 & 0,7 & 0,7 & 1,1 \\
\hline Cuba & 0,6 & 0,8 & 0,8 & 0,9 \\
\hline Ecuador & 0,8 & 1,2 & 1,0 & 2,2 \\
\hline Uruguay & 1,2 & 1,4 & 1,1 & 1,2 \\
\hline Venezuela & 1,0 & 0,9 & 1,0 & 0,6 \\
\hline Marruecos & 1,1 & 1,1 & 1,1 & 1,7 \\
\hline Mauritania & 1,4 & 1,8 & 1,5 & 2,4 \\
\hline China & 1,5 & 1,8 & 1,4 & 2,5 \\
\hline India & 2,4 & 2,0 & 2,6 & 2,9 \\
\hline
\end{tabular}

Fuente: Padrón continuo de población. INE. Elaboración propia. 
En segundo lugar, hay que destacar el hecho de que en algunas zonas, y para algunas nacionalidades, se ha desarrollado una actividad inmobiliaria específica, desvinculada de los enclaves turísticos de masas. El caso más representativo es el de los alemanes en la isla de Tenerife. A partir de la importancia que ha tenido y tiene el centro turístico de Puerto de la Cruz, al norte de la isla, en el mercado alemán, se ha desarrollado una importante actividad inmobiliaria destinada a este mismo mercado en los municipios aledaños (Los Realejos, La Orotava, La Matanza de Acentejo, Santa Úrsula, El Sauzal, Los Silos, Icod de los Vinos), hasta tal punto que la localización de los alemanes en Tenerife es completamente opuesta a la del resto de las nacionalidades europeas. Los alemanes residen masivamente en el norte, en espacios «rururbanos», ricos en vegetación y con un menor número de horas de insolación directa, mientras que el resto de los europeos prefieren el sur insular, donde la temperatura y el sol son el principal reclamo (véase la figura 11).

Pero además, y como tercer factor, las disparidades en la localización también atienden al modo en que se ha desarrollado el mercado turístico-residencial

Figura 11

Coeficientes de localización de los alemanes por sección en 2006

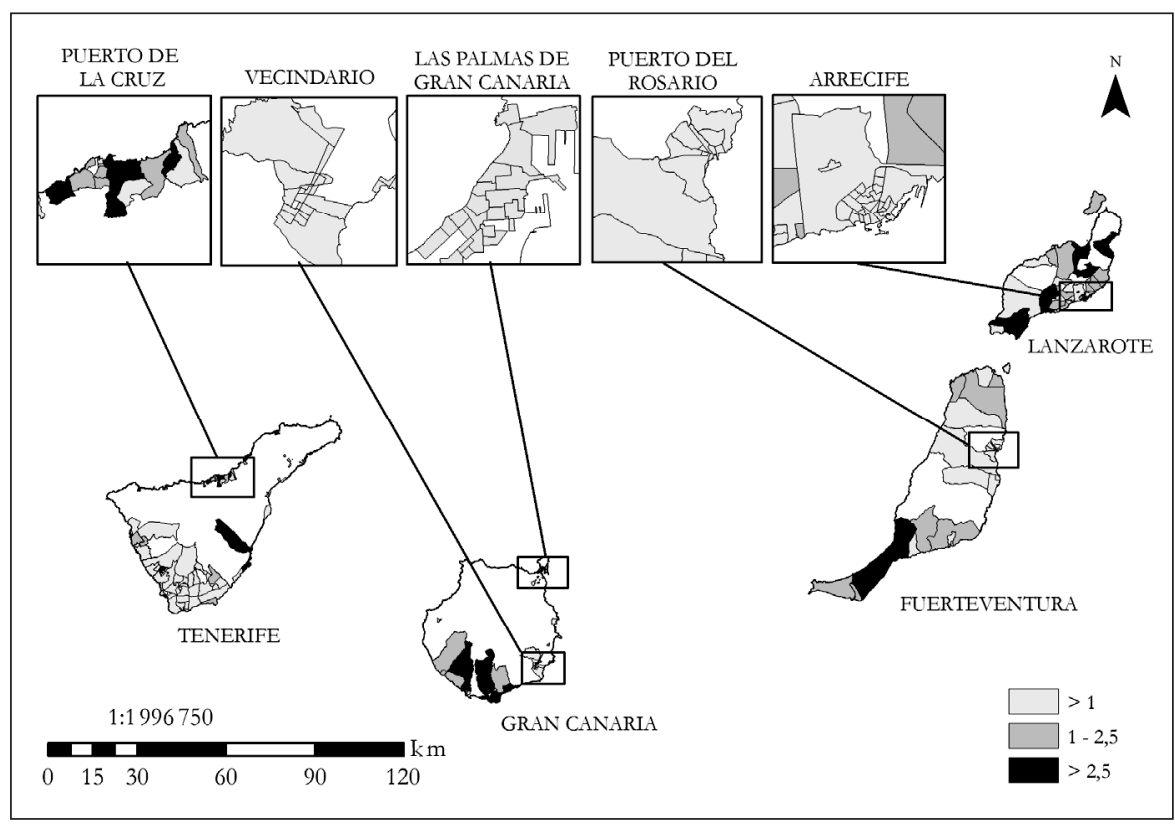

Fuente: Padrón continuo de población. INE. Elaboración propia. 
Figura 12

Coeficientes de localización de los británicos por sección en 2006

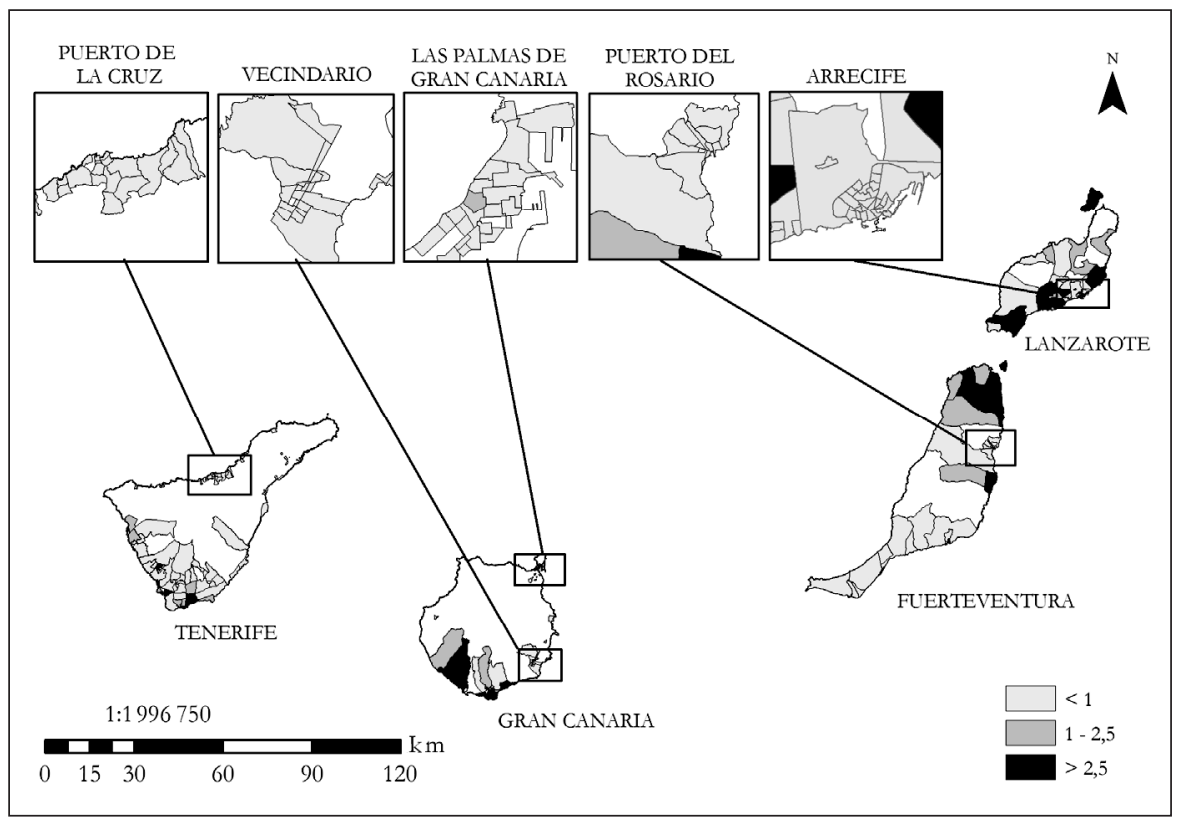

Fuente: Padrón continuo de población. INE. Elaboración propia.

en Canarias. Existe una especialización según la nacionalidad de la actividad inmobiliaria en las áreas turístico-residenciales. Por ejemplo, en Gran Canaria, las secciones turísticas del municipio de Mogán se han destinado al mercado británico, mientras que en el otro gran municipio turístico del sur insular, San Bartolomé de Tirajana, la actividad promotora se orienta mucho más al mercado alemán. El ejemplo se reproduce, incluso de un modo más drástico en Fuerteventura. En esta isla, las dos nacionalidades con mayor presencia, alemanes y británicos, se ubican en el sur (Pájara) y norte y centro (La Oliva y Antigua), respectivamente. Y qué decir de Tenerife, donde los franceses destacan en algunas secciones de Adeje y Guía de Isora en el sur y oeste de la isla, los británicos en San Miguel de Abona, Adeje, Arona y Santiago del Teide en el sur y oeste de la isla y los alemanes en Arico, al este (véase la figura 12). Pero es que además, en las secciones eminentemente turísticas, es frecuente que se aprecie una cierta especialización según la nacionalidad, salvo en la isla de Lanzarote, donde no se detectan relaciones significativas. 
En otro orden de cosas, el carácter terciarizado de la economía canaria ha supuesto una fuerte polarización del mercado de trabajo, con una gran cantidad de mano de obra de bajo estatus salarial. Este hecho, unido al desequilibrio entre la mano de obra local y el crecimiento económico de las islas, ha generado importantes oportunidades de empleo para los inmigrantes, como ya ha sido descrito para otras zonas (Sassen, 1991; Kempen, 2007). Si esto lo vinculamos a la falta de oportunidades y a las malas condiciones de vida que sufre la población en muchas zonas del planeta, se comprende que en los últimos años haya crecido el número de extranjeros que llegan a las islas por motivos laborales. En este flujo de inmigrantes laborales hay que incluir al resto de las nacionalidades consideradas.

La motivación laboral de estos flujos migratorios repercute en que su localización territorial esté determinada por las oportunidades de los mercados de trabajo insulares. Por este motivo, turismo y servicios urbanos son los dos factores de localización esenciales y la tendencia de los grupos considerados ha sido vivir lo más cerca posible de los mercados de trabajo. En la isla de Tenerife se aprecia, como en ninguna otra, la vinculación entre dinamismo económico e inmigración. En Tenerife las zonas económicamente emergentes se sitúan en las áreas turísticas del suroeste y sureste, motivo por el que la inmigración laboral en el norte de la isla, a pesar de la mayor concentración urbana y de la presencia del importante núcleo turístico de Puerto de la Cruz, tiene un peso relativo reducido.

Existen, no obstante, algunas diferencias suficientemente importantes en la posición que ocupa cada nacionalidad en el mercado de trabajo, como para que este hecho tenga un correlato en su localización. El caso de la comunidad rumana es paradigmático. Buena parte de ella se emplea en la zafra del tomate de exportación, por lo que la presencia de este colectivo es significativa en algunas secciones de los municipios donde se desarrolla esta actividad, como es el caso de la Aldea de San Nicolás, al oeste de Gran Canaria, o en el municipio de Arico, situado al este de Tenerife. El empleo en la agricultura del plátano, en el municipio de Icod de los Vinos, en el norte de Tenerife, es, igualmente, el factor que determina la localización de un importante de grupo de trabajadores rumanos en esta zona.

Además, el análisis a nivel de sección nos revela que la localización de los inmigrantes laborales depende de otros factores, entre los que cabe citar el mercado de la vivienda y las características culturales y de grupo de algunas nacionalidades.

En España, la política de vivienda no ha permitido intervenir adecuadamente en los mercados habitacionales, por lo que desde 1998 ha ido creciendo 
el esfuerzo familiar para acceder a una vivienda. En este contexto general, los inmigrantes han sido un grupo social especialmente vulnerable. Son muy pocos los que han podido acceder a la vivienda protegida, tanto por ser extranjeros como por la desinformación que tienen respecto de las ayudas públicas, y a ello se suma el hecho de que el acceso a la escasa vivienda protegida lo ha sido en España en régimen de propiedad, lo que exige una cierta capitalización o garantías laborales, de las que ellos carecen. Por todo esto, la localización de los extranjeros se explica por las oportunidades que el mercado de la vivienda libre ofrece $y$, en especial, por las que brinda el mercado de alquiler.

De este modo, en las principales capitales insulares estos colectivos residen lo más cercano posible a sus puestos de trabajo, allí donde se encuentran las viviendas en alquiler a unos precios adecuados. El caso de Las Palmas de Gran Canaria es muy significativo. La mayor parte de estos colectivos habitan en el entorno del Puerto de la Luz y Playa de las Canteras, donde se han puesto en alquiler muchas viviendas, unas reconvertidas a partir de antiguos complejos extrahoteleros y otras relacionadas con el abandono de inmuebles por parte de la población local, debido al proceso de degradación ambiental y social que se ha dado en buena parte de este sector. Este hecho ya ha sido mencionado para otras muchas ciudades europeas y americanas y, como en muchos de estos espacios, los extranjeros vienen a llenar un vacío ya existente y no tanto a competir con la población local.

Además, se da la peculiaridad de que algunas zonas turísticas de Canarias no han reservado espacio, o si acaso muy poco, para las funciones residenciales de la población trabajadora, lo que ha determinado que los colectivos de inmigrados tengan que residir en municipios distantes de sus puestos de trabajo. La isla de Gran Canaria nos brinda el mejor ejemplo en este sentido; en ella la población extranjera trabajadora se localiza, además de en algunas secciones residenciales colindantes a las zonas turísticas, en otras del municipio de Santa Lucía de Tirajana, al este de la isla, y en los municipios aledaños de Agüimes e Ingenio, ante la dificultad de competir por las escasas viviendas en alquiler de los municipios turísticos del sur de la isla. Una situación similar se detecta en Lanzarote, donde salvo algunas secciones residenciales, en el entorno del área turística de Puerto del Carmen, la mayor parte de los inmigrantes, a pesar de estar empleados en las actividades y zonas turísticas de la isla, vive en Arrecife y San Bartolomé, municipios no turísticos.

La fase del proceso migratorio o las peculiaridades de algunas nacionalidades tienen también cierta incidencia en la distribución de los inmigrantes laborales. Los miembros de los colectivos con una mayor presencia en la islas y, por tanto, con un proceso migratorio de más larga duración, tienden a 
diversificar las actividades económicas en las que se emplean, como resultado de una mayor integración en los mercados laborales insulares. En consecuencia, sus niveles de concentración son bajos. En esta situación cabe reconocer a marroquies, argentinos, cubanos, venezolanos y colombianos. Los primeros, por ejemplo, se emplean en un cierto tipo de comercio (de marroquinería), en el propio mercado de contratación agrícola, en el sector turístico, en el sector de la construcción..., motivo por el que están presentes en muchas secciones de los espacios de la inmigración (véanse las figuras 13 y 14). Por el contrario, los ecuatorianos y los bolivianos, con un ciclo migratorio más reciente, se muestran menos integrados en las economías insulares y más dependientes de las relaciones intragrupales.

En otros casos, son las características sociodemográficas y culturales de los inmigrantes las que determinan su presencia territorial. Así, por ejemplo, la localización de los chinos está condicionada por su tendencia a residir junto a sus negocios, generalmente orientados a la restauración y al comercio, así como por su fuerte conciencia de grupo. En sentido opuesto se manifiestan

Figura 13

Coeficientes de localización de los marroquíes por sección en 2006

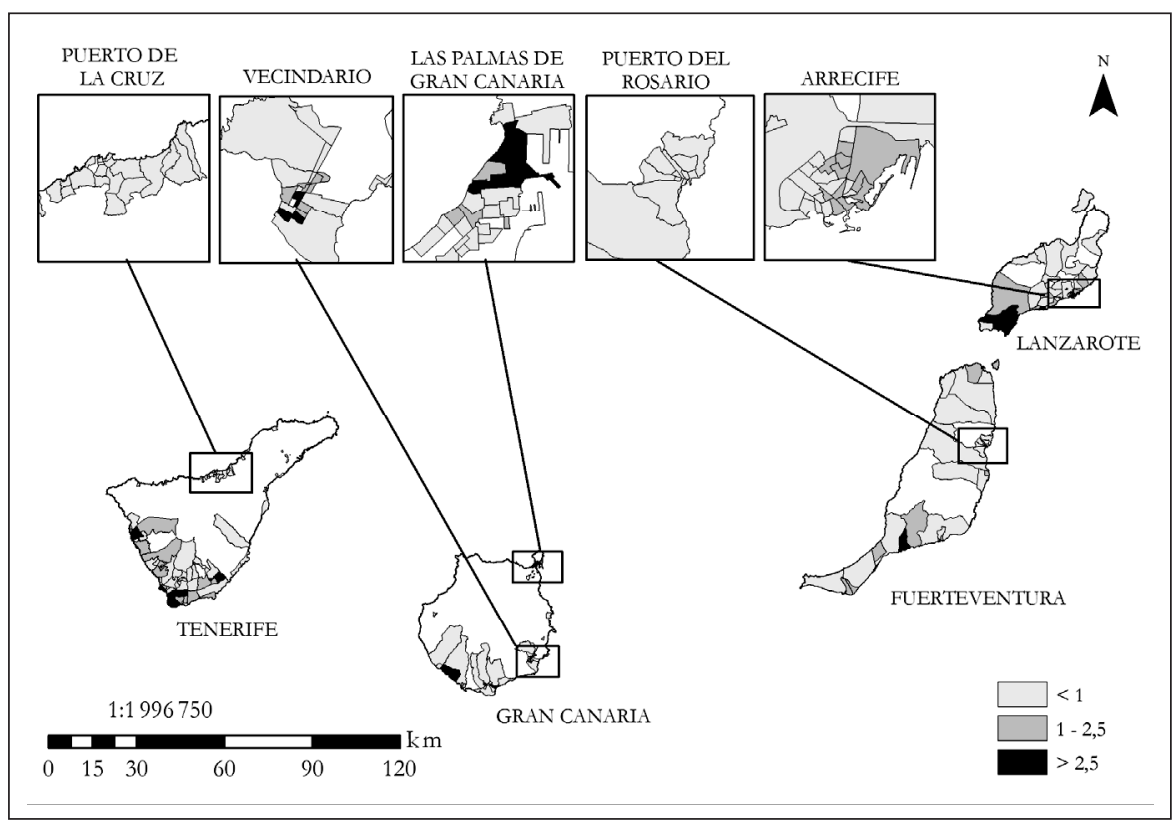

Fuente: Padrón continuo de población. INE. Elaboración propia.

Investigaciones geográficas, n. ${ }^{\circ} 45$ (2008) 
Figura 14

Coeficientes de localización de los colombianos por sección en 2006

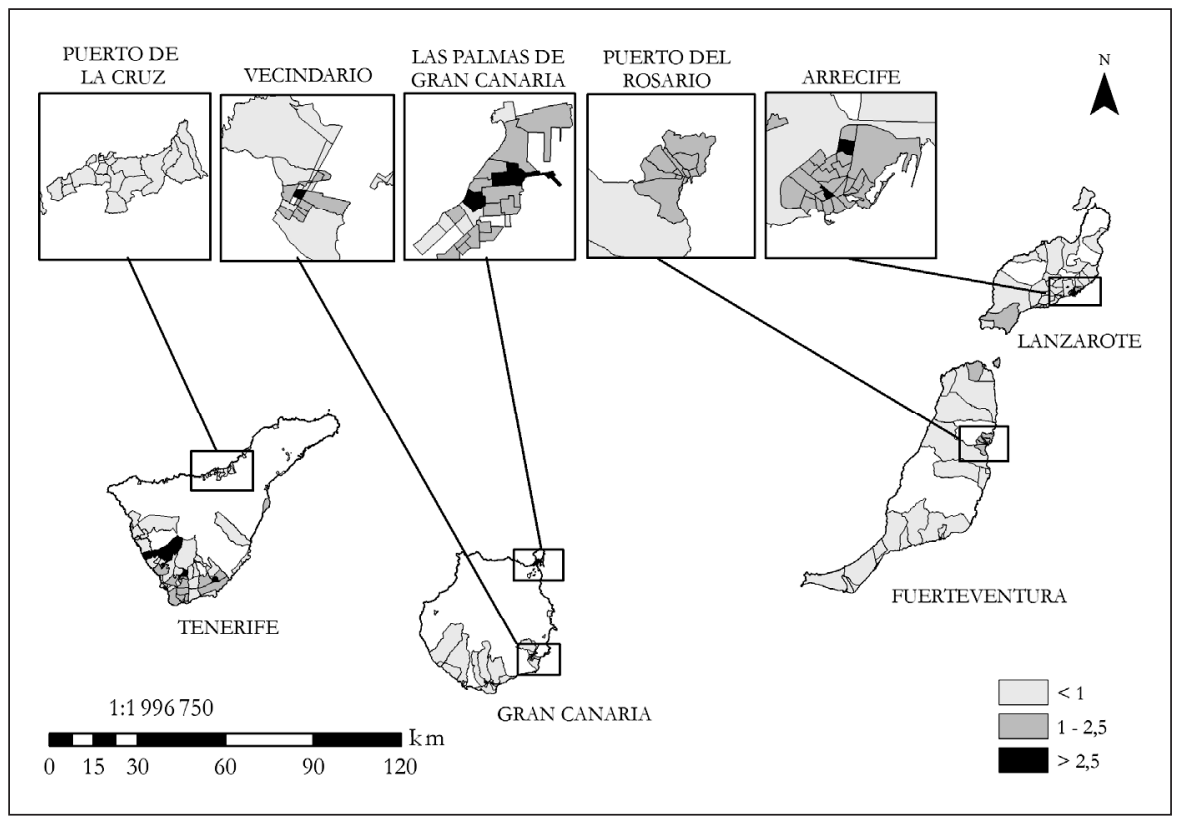

Fuente: Padrón continuo de población. INE. Elaboración propia.

las comunidades argentina, venezolana y cubana, que refuerzan su tendencia a la dispersión a causa de su gran heterogeneidad social, más próxima a la propia estructura social de la población española, en el primer caso, y a los lazos tradicionales que Canarias ha mantenido con las dos segundas.

Además de las tendencias territoriales y de los factores de localización, el microanálisis permite apreciar que, frecuentemente, existe una cierta especialización residencial de la sección en función de la nacionalidad para el caso de la inmigración laboral. En otros términos, en muchos casos, las nacionalidades tienden a vivir en secciones diferentes del mismo municipio. Este hecho se manifiesta en las bajas tasas de correlación bivariadas que se registran entre las nacionalidades consideradas, en algunos casos incluso de carácter inverso. Salvo excepciones notables, como la coincidencia espacial que se registra entre colombianos y ecuatorianos en Lanzarote y Fuerteventura, o argentinos y colombianos en Tenerife, se dibuja una cierta tendencia a la segregación espacial por nacionalidad. A pesar de estos datos, ¿podemos concluir que existe segregación por nacionalidad? 


\section{Segregación de la población extranjera y especialización territorial}

El comportamiento territorial, como ya hemos señalado, indica una superposición de los diferentes modelos de distribución de los extranjeros residentes en Canarias, lo que nos hace reconocer determinadas secciones en las que la mayor parte de la población extranjera comparte la misma nacionalidad, y otras, en las que la mezcla multicultural es su principal característica. En otras palabras, podemos encontrar secciones especializadas en un flujo migratorio determinado y otras con una baja especialización.

Desde una aproximación global, pero ilustrativa de este fenómeno, se pueden diferenciar dos grandes perfiles migratorios en las islas: el constituido por los naturales de la Unión Europea ${ }^{7}$ y otros países del centro y norte de Europa y el formado por el resto de las nacionalidades. Para confrontar ambos contingentes, a escala de sección e isla, hemos empleado el índice de localización, lo que nos ha permitido clasificar las secciones en cuatro categorías: altamente especializadas en población europea (el índice es superior a 1,4 para este contingente), altamente especializadas en población no europea (un índice superior a 1,2) y ligeramente especializadas en población europea o no europea, con índices de localización entre 1 y 1,4 en el primer caso y 1 y 1,2 en el segundo. La expresión cartográfica de estos indicadores revela un mosaico de realidades territoriales muy diferentes (figura 15). Como cabía esperar, la mayor parte de las secciones turísticas presentan una especialización ligera o elevada en el perfil migratorio europeo. El sur de Gran Canaria y la casi totalidad de la isla de Lanzarote son los mejores ejemplos. No obstante, la heterogeneidad territorial es mayor de lo esperado y, así, las secciones turísticas del sur de Tenerife presentan una ligera especialización no europea.

Los ámbitos urbanos están ligera o altamente especializados en el perfil migratorio no europeo. Las Palmas de Gran Canaria y Vecindario en Gran Canaria; Puerto del Rosario y otros núcleos del sur de la isla en Fuerteventura; Arrecife en Lanzarote y algunos enclaves urbanos del sur de Tenerife registran los mayores índices de especialización.

El hecho de que algo más del $40 \%$ de las secciones tenga una alta especialización en alguno de los dos perfiles migratorios parece aventurar que podemos encontrarnos con realidades segregadas desde el punto de vista territorial.

7. Recuérdese que en la fecha de referencia en este estudio, en 2006, aún no se había producido la ampliación de la Unión Europea a los 27 miembros que la conforman en la actualidad. 
La segregación se puede definir a partir de dos ópticas, la sociológica y la geográfica (White, 1983). En el primer caso existe segregación cuando hay ausencia de interacción entre grupos sociales. Sin embargo, la segregación geográfica o residencial depende de la distribución de los grupos sociales en el espacio, de tal modo que podemos relacionar concentración con segregación (Brun y Rhein, 1994). Consecuentemente, la segregación residencial se relaciona con la sobrerrepresentación de un grupo, en un área determinada, a una escala precisa. No es necesario que coexistan situaciones de segregación definidas desde un punto de vista geográfico y sociológico, pero ciertamente suelen coincidir, en la medida en que las estructuras espaciales reflejan las sociales.

Pues bien, nos ha parecido oportuno estimar cuantitativamente la segregación residencial en Canarias mediante el índice sintético de segregación, un índice que relaciona la población total y por nacionalidad de la sección con la población total y por nacionalidad de la isla.

Figura 15

Especialización según el perfil migratorio por secciones en 2006

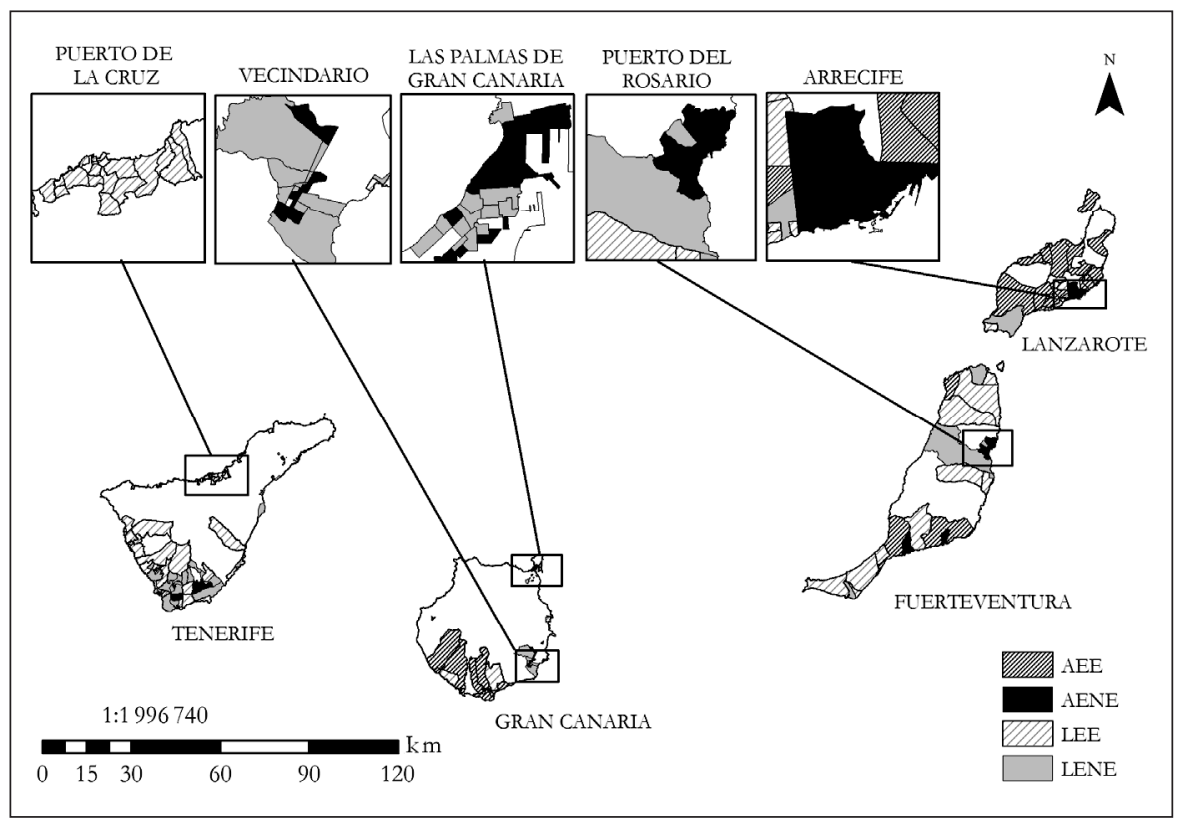

Fuente: Padrón continuo de población. INE. Elaboración propia. 


\section{Cuadro 2}

Índices de segregación por islas y nacionalidad

\begin{tabular}{|l|c|c|c|c|}
\hline & Fuerteventura & Gran Canaria & Lanzarote & Tenerife \\
\hline Alemania & 0,54 & 0,58 & 0,54 & 0,40 \\
\hline Bélgica & 0,42 & 0,53 & 0,47 & 0,43 \\
\hline Francia & 0,43 & 0,41 & 0,48 & 0,38 \\
\hline Italia & 0,36 & 0,29 & 0,35 & 0,34 \\
\hline Países Bajos & 0,47 & 0,54 & 0,50 & 0,33 \\
\hline Portugal & 0,27 & 0,33 & 0,29 & 0,36 \\
\hline Reino Unido & 0,63 & 0,56 & 0,57 & 0,48 \\
\hline Rumanía & 0,46 & 0,45 & 0,38 & 0,42 \\
\hline Argentina & 0,26 & 0,29 & 0,30 & 0,28 \\
\hline Bolivia & 0,71 & 0,63 & 0,55 & 0,72 \\
\hline Colombia & 0,29 & 0,30 & 0,31 & 0,38 \\
\hline Cuba & 0,22 & 0,29 & 0,24 & 0,32 \\
\hline Ecuador & 0,34 & 0,45 & 0,42 & 0,52 \\
\hline Uruguay & 0,45 & 0,44 & 0,35 & 0,41 \\
\hline Venezuela & 0,31 & 0,33 & 0,33 & 0,24 \\
\hline Marruecos & 0,40 & 0,41 & 0,37 & 0,46 \\
\hline Mauritania & 0,54 & 0,57 & 0,46 & 0,61 \\
\hline China & 0,52 & 0,51 & 0,55 & 0,62 \\
\hline India & 0,65 & 0,68 & 0,70 & 0,69 \\
\hline
\end{tabular}

Fuente: Padrón continuo de población. INE. Elaboración propia.

Sin embargo, se ha de considerar que hemos aplicado el índice sintético de segregación a un ámbito espacial discontinuo (los territorios de la inmigración), lo que no nos informa del nivel de segregación con el que una determinada nacionalidad se localiza en la isla, ya que no se ha considerado el conjunto de las secciones insulares ${ }^{8}$. No obstante, los resultados estadísticos que arroja este índice son significativos y pueden apreciarse en el cuadro 2. Si consideramos 0,6 como el límite que define una situación de segregación (Masey, 1985), esto sólo sucede en el caso de los hindúes en todas las islas, bolivianos en Fuerteventura, Gran Canaria y Tenerife; chinos y mauritanos en Tenerife y británicos en Fuerteventura. Además, hindúes, chinos, británicos, alemanes, belgas, holandeses, bolivianos y ecuatorianos superan un índice de 0,5 . Por tanto, en términos generales el nivel de segregación no es elevado.

8. Sabemos que la inmigración en Canarias reside en determinados espacios y de haber considerado la totalidad de las secciones insulares los índices de segregación hubieran sido siempre altos 
Pese a las diferencias de escala y de ámbitos de referencia, la comparación con otras zonas en las que se ha aplicado el índice sintético de segregación muestra una situación similar en los casos de Barcelona (Martori, Hoberg y Surinach, 2006; Bayona i Carrasco, 2007) y en los análisis realizados para otros ámbitos urbanos europeos (Petsimeris, 1998; Malheiros, 2002; Musterd y Deurloo, 2002). En líneas generales, las peculiaridades de Canarias vienen dadas por los altos valores registrados por los asiáticos (especialmente llamativo en el caso de los chinos), por algunas nacionalidades de reciente instalación como bolivianos o ecuatorianos y, especialmente, por la mayor segregación que se registra entre las comunidades del norte y centro de Europa.

En los distintos estudios que se han llevado a cabo, para abordar el fenómeno de la segregación, destacan como factores condicionantes la diferenciación social y la desigualdad en el valor del espacio (Ocaña, 2005). En relación con el primer aspecto, se encuentran el estatus económico de los inmigrantes y la relación de sus recursos con el precio de la vivienda (Leal, 2002), el nivel de integración según nacionalidad y tiempo de asentamiento, así como la puesta en práctica de tendencias a la congregation o auto-exclusión (Amersfoort y De Klerk, 1987). En otras palabras, se trata de las diferencias de los inmigrantes en relación con sus recursos económicos, sociales, culturales y políticos. En relación con el segundo aspecto, hallamos la particular distribución espacial de estructuras territoriales y la puesta en práctica de políticas públicas de intervención (Kempen y Ozüekren, 1998).

Estos factores se reconocen en Canarias y en toda España, ya que el archipiélago comparte con el resto del territorio nacional la falta de intervención pública en el sector de la vivienda, con un nivel que se sitúa por debajo del estándar europeo, en lo que concierne a las políticas que definen el estado del bienestar. También comparte un mercado de la vivienda rígido, caracterizado por la preponderancia absoluta de la compra como régimen de tenencia, y una elevada ratio entre el precio de los inmuebles y los recursos de los hogares, factores todos ellos que favorecen la existencia de segregación geográfica. De igual modo, reconocemos que la diversidad de estructuras urbanas determina diferentes niveles de segregación. En el sur de Gran Canaria, la planificada separación entre suelo turístico y suelo residencial favorece unos mayores niveles de diferenciación residencial frente a lo que sucede en otras islas, como Tenerife, donde cohabitan de un modo más orgánico ambos usos. Por último, el desarrollo de espacios urbanos fracturados (Marcuse, 1993) está presente en las grandes capitales insulares como en cualesquiera otros espacios urbanos.

Ahora bien, a tenor de los resultados estadísticos obtenidos, se demuestra que las dos variables que definen básicamente la segregación por nacionalidad 
en el archipiélago son la tendencia a la autoexclusión de algunos colectivos que, sin embargo, no llegan a definir mayoritariamente gated communities, como se advierte en el caso de alemanes, británicos, franceses, belgas, holandeses e hindúes y el carácter incipiente del proceso migratorio de algunas nacionalidades, tales como bolivianos, ecuatorianos, mauritanos y chinos.

Por consiguiente, en Canarias estamos hablando de un proceso de segregación estructural, inherente al propio fenómeno inmigratorio, en el primer caso, y de otro coyuntural, que podrá confirmarse a medida que se consoliden estos flujos, como ha podido comprobarse en el caso de Barcelona (Bayona i Carrasco, 2007). No faltan, no obstante, estudios que niegan este carácter coyuntural, como se ha afirmado para los inmigrantes africanos en el campo almeriense, donde la movilidad y mejora residencial no parece que dependa tanto de la mejora económica y del tiempo de permanencia de los inmigrantes sino de factores sociales que van a actuar en sentido opuesto, como la discriminación por parte de los propietarios de inmuebles (Checa y Arjona, 2006).

\section{Conclusiones}

1. Los procesos de diferenciación social del espacio, desde la perspectiva de la extranjería, adquieren una nueva dimensión interpretativa cuando se emplean en su análisis la óptica microescalar y la herramienta estadística que representan los datos georreferenciados de un sistema de información.

2. La inmigración ha tenido un crecimiento muy concentrado en el tiempo en el tránsito de los siglos XX al XXI, en Canarias y en el conjunto de España, lo que representa una situación excepcional en el contexto de los países de la Unión Europea.

3. El fenómeno migratorio, en clara sintonía con su carácter reciente, intenso y dinámico, queda aún indefinido en muchas de sus facetas geográficas.

4. Desde la perspectiva de la localización espacial de los extranjeros en Canarias, el análisis de microescala revela un comportamiento muy alejado de la normalidad estadística, lo que exige establecer una selección de aquellas secciones censales en que la extranjería alcanza un umbral mínimo. Éstas se circunscriben, principalmente, a las áreas de mayor especialización turística, las que definimos como «espacios de la inmigración», frente a las que no registran una significativa presencia de población extranjera, es decir, que se comportan como «espacios de repulsión».

5. Además de los factores vinculados a la actividad turística, otros de orden secundario explican también la distribución territorial de la extranjería: los procesos de urbanización y de las funciones a ellos asociadas (comerciales, portuarias, servicios personales), la contratación de mano de obra extranjera 
en la agricultura de exportación y el retorno de emigrantes o descendientes a ciertos ámbitos urbano-turísticos.

6. En los últimos años se han diversificado los factores de atracción residencial, por el creciente número de actividades que conforman un mercado laboral más diversificado y por el proceso de articulación e integración de los espacios económico-residenciales a escala insular, lo que ha representado una evolución en el modelo territorial de la extranjería hacia una mayor homogeneidad.

7. Un análisis de detalle de la distribución territorial nos permite apreciar patrones territoriales diferentes en función de la nacionalidad, que se pueden sintetizar en dos tipologías migratorias, una de carácter turístico-laboral (alemanes, británicos...), con una mayor tendencia a la concentración, y otra estrictamente laboral, que se corresponde con una mayor dispersión (italianos, portugueses, argentinos, colombianos, venezolanos y cubanos), lo que nos hace deducir la desigual incidencia de los factores de localización en función de la nacionalidad y del propio marco insular.

8. De forma complementaria, la distribución está condicionada por otras causas, como la especialización de la actividad inmobiliaria en las áreas turístico-residenciales según la nacionalidad: alemanes en el norte de Tenerife, británicos en el oeste de Gran Canaria... o por el propio dinamismo del mercado laboral, lo que explica que en Tenerife, por ejemplo, la inmigración se localice en las zonas económicamente emergentes del suroeste y sureste insulares o que el colectivo de rumanos resida, mayoritariamente, en las secciones censales donde hay agricultura de exportación.

9. El mercado de la vivienda, un mercado rígido, caracterizado por la preponderancia absoluta de la compra como régimen de tenencia, y una elevada ratio entre el precio de los inmuebles y los recursos de los hogares, hace que muchos de los extranjeros residan allí donde predomina la vivienda en alquiler, un factor adicional, por tanto, en la interpretación de su distribución territorial.

10. Por último, la fase del proceso migratorio y los factores grupales tienen cierta incidencia en la distribución de los inmigrantes laborales. Los que se relacionan con un proceso migratorio de más larga duración tienden a diversificar las actividades económicas en las que se emplean. En consecuencia, sus niveles de concentración son bajos, mientras que los de ciclo migratorio más reciente se muestran menos integrados en las economías insulares y más dependientes de las relaciones intragrupales.

11. El índice sintético de segregación por nacionalidad en los espacios de la inmigración tiene unos valores bajos, sin embargo se aprecia una ligera 
tendencia a la autoexclusión de algunos colectivos (alemanes, británicos, franceses, belgas, holandeses e hindúes) y el carácter incipiente del proceso migratorio de algunas otras nacionalidades que alcanzan, también, las cifras más altas (bolivianos, ecuatorianos, mauritanos y chinos). Por consiguiente, en Canarias estamos hablando de un proceso de segregación estructural, inherente al propio fenómeno inmigratorio, en el primer caso, y de otro coyuntural, que podrá confirmarse o no a medida que se consoliden estos flujos.

\section{Bibliografía}

AldREY VÁzQUEZ, J.A. (2005): «El uso de fuentes demográficas a escala microespacial y su aplicación al estudio de un territorio: la mitad sur de la provincia de A Coruña» en Cuadernos Geográficos 36, pp. 507-515.

AMERSFOORT, H. VAN y L. DE KLERK (1987): «The dynamics of immigrant settlement: Surinamese, Turks and Moroccans in Amsterdam 1973-1983» en G. GLEBE y J. O'LOUGHLIN (eds.): Foreign minorities in continental European cities, pp. 199-222, Stuttgart, Franz Steiner Verlag, 296 pp.

BASSETT, K. Y J. SHORT (1980): Housing and residential structure. Alternative approaches, London, Routledge and Kegan Paul, 254 pp.

BAYONA I CARRASCO, J. (2007): «La segregación residencial de la población extranjera en Barcelona: ¿Una segregación fragmentada?» en Scripta Nova, XI, 235, 15 de marzo, [en línea], <http://www.ub.es/geocrit/sn/sn-235.htm>. [Consulta: 10/01/2008].

BERRY, B.J.L. y J.D. KASARDA (1977): Contemporary urban ecology, Nueva York, Macmillan, 498 pp.

BRUN, J. y C. RHEIN (1994): La ségrégation dans la ville: concepts et mésures, París, L'Harmattan, $2 \mathrm{v}$.

CHECA Olmos, J.C. y A. ARJONA GARRIDO (2006): «Segregación y condiciones residenciales de los inmigrantes africanos en Almería (España)» en Migraciones Internacionales, 3, 3, pp. 81-106.

COMItÉ DE EXPERTOS SOBRE Población E INMIGRACIÓN EN CANARIAS (2003): Informe sobre población e inmigración en Canarias, Las Palmas de Gran Canaria, Gobierno de Canarias, 276 pp.

Domínguez MujiCA, J. (2006): «La inmigración extranjera en Canarias en el cambio de siglo» en Estudios Geográficos LXVII, pp. 471-494.

-, R. Díaz Hernández y J.M. ParReño Castellano (2001): Inmigración extranjera e integración. La inmigración irregular en Canarias (1999-2000), Las Palmas de Gran Canaria, Gobierno de Canarias, 249 pp.

- y J.M. Parreño Castellano (2005): «Los Sistemas de Información Corporativos y su utilización para el análisis socio-demográfico: una propuesta metodológica» en Cuadernos Geográficos 36, pp. 91-103. 
Domínguez Mujica, J., J.M. Parreño Castellano y T. Pérez García (2006): Un Sistema de Información Geodemográfica: vivienda y población en el barrio de Escaleritas. Las Palmas de Gran Canaria, Las Palmas de Gran Canaria: Ayuntamiento de Las Palmas de Gran Canaria, [en línea], <http://geoportal.laspalmasgc.es/sicam_doc/geodemografia/index.html>. [Consulta: 10/01/2008].

DunCAN, O.D. y A. DUNCAN (1955): «A methodological analysis of segregation indexes» en American Sociological Review, vol. 41, pp. 210-217.

KEMPEN, R. VAN y A.S. ÖZÜEKREN (1998): «Ethnic segregation in cities: new forms and explanations in a dynamic world» en Urban Studies, 35, 10, pp. 1631-1656.

KEMPEN, R. VAN (2007): «Divided cities in the $21^{\text {st }}$ century: challenging the importance of globalisation» en Journal of Housing Built Environ, 22, pp. 13-31.

Kesteloot, C. (1986): «Concentración d'étrangers et politique urbaine à Bruxeles» en Revue Européenne des Migrations Internationales, vol. 2, n. ${ }^{\text {o }} 3$, pp. 151-167.

LEAL, J. (2002): «Segregación social y mercados de vivienda en las grandes ciudades» en Revista Española de Sociología, 2, pp. 59-75.

MACKENZIE, R. (1925/1967): «The ecological approach to the study of the human community» en R. PARK., E. BurgesS y R. MACKEnZIE (eds.): The City, Chicago, University of Chicago Press, 239 pp.

MAlheiros, J. (2002): «Ethnic-cities: residential patterns in the Northern European and Mediterranean metropolises. Implications for policy design» en International Journal of Population Geography, 8, pp. 107-134.

MARCUSE, P. (1993): «What's so new about divided cities» en International Journal of Urban and Regional Research, 17, 3, pp. 355-365.

MARTORI, J.C. y K. HOBERG (2004) «Indicadores cuantitativos de segregación residencial. El caso de la población inmigrante en Barcelona» en Scripta Nova, VIII, 169, 15 de julio, [en línea], <http://www.ub.es/geocrit/sn/sn-169.htm>. [Consulta: 10/01/2008].

MARTORI, J.C., K. Hoberg y J. SURINACH (2006): «Población inmigrante y espacio urbano. Indicadores de segregación y pautas de localización» en EURE, 32, 97, [en línea], <http://www.scielo.cl/scielo.php?script=sci_arttext\&pid=S025071612006000300004\&lng=es\&nrm=iso>. [Consulta: 10/01/2008].

MASSEY, D.S. y N.A. DENTON (1988): «The dimensions of residential segregation» en Social Forces, 67, pp. 281-315.

MorRILL, R.L. (1991): «On a measure of geographical segregation» en Geography Research Forum, 11, pp. 25-38.

Musterd, S. y R. Deurloo (2002): «Unstable immigrant concentration in Amsterdam: Spatial segregation and integration of newcomers» en Housing Studies, 17, pp. 487-503. 
OCAÑA OCAÑA, C. (2005): «Microanálisis sociodemográfico de espacios urbanos» en Boletín de la A.G.E., 40, pp. 5-34.

PEACH, C. (1975): «Immigrants in the inner city» in Geographical Journal, 141, 3, pp. 372-379.

PETSIMERIS, P. (1998): «Urban decline and new social and ethnic divisions in the core cities of the Italian industrial triangle» en Urban Studies, 35, pp. 449-465.

REX, J. y R. MOORE (1967): Race, community and conflict, Londres, Oxford University Press, $304 \mathrm{pp}$.

SABATINi, F. G. CÁCERES y J. CERDA (2001): «Segregación residencial en las principales ciudades chilenas: tendencias de las tres últimas décadas y posibles cursos de acción» en EURE, 27, 82, [en línea], <http://www.scielo.cl/scielo. php?script=sci_arttext $\&$ pid=S0250-71612001008200002\&lng=es\&nrm $=\mathrm{i}$ so>. [Consulta: 10/01/2008].

SASSEn, S. (1991): The global city: New York, London, Tokio; Princeton, NJ; Priceton University Press; 447 pp.

SHEVKY, E. y W. BELL (1955): Social area analysis: theory, illustrative application and computational procedures, Stanford, Stanford University Press, $70 \mathrm{pp}$.

WACQuAnt, L. (2001): Parias urbanos. Marginalidad en la ciudad a comienzos del mileno, Buenos Aires, Manantial, 208 pp.

White, M. (1983): «The measurement of spatial segregation» en American Journal of Sociology, 88, 5, pp. 1008-1018.

WONG, D.W. (1993): «Spatial indices of segregation» en Urban Studies, 30, pp. 559-572.

WONG, D.W. (2005): «Formulating a general spatial segregation measure» en The Professional Geographer, 57, 2, pp. 285-294. 